\title{
Simulation and Analysis of the Complex Dynamic Behavior of Supply Chain Inventory System from Different Decision Perspectives
}

\author{
Zusheng Zhang $\mathbb{D}^{1,2}$ Xu Wang $\mathbb{D}^{1,}{ }^{1,2}$ Shanshan Yang, ${ }^{3}$ Yingbo $W u\left(\mathbb{D},{ }^{4}\right.$ and Jianhui Du ${ }^{1,2}$ \\ ${ }^{1}$ College of Mechanical Engineering, Chongqing University, Chongqing, China \\ ${ }^{2}$ Chongqing Key Laboratory of Logistics, Chongqing University, Chongqing, China \\ ${ }^{3}$ School of Economics and Business Administration, Chongqing University, Chongqing, China \\ ${ }^{4}$ School of Big Data \& Software Engineering, Chongqing University, Chongqing, China \\ Correspondence should be addressed to Xu Wang; wx921@163.com
}

Received 27 May 2020; Revised 18 July 2020; Accepted 3 August 2020; Published 30 September 2020

Guest Editor: Baltazar Aguirre-Hernández

Copyright (c) 2020 Zusheng Zhang et al. This is an open access article distributed under the Creative Commons Attribution License, which permits unrestricted use, distribution, and reproduction in any medium, provided the original work is properly cited.

\begin{abstract}
Based on the local decision perspective and the global decision perspective, considering the limitation of supply capacity and prohibiting returns, the system dynamics method is used to establish a nonlinear supply chain system model. We use the $Z$ transform theory to transform the dynamic transfer equation into a block diagram, build a supply chain system simulation model, and use it to conduct simulation experiments. The Wolf reconstruction method is used to calculate the largest Lyapunov exponent (LLE) value of each node or combined system to judge the stability of the system. Based on different decision-making perspectives, under different combinations of safety stock factors and demand scenarios, the adjustment coefficients' decision-making schemes that keep each node in a stable state are obtained. Then, we comparatively analyze the inventory changes of each node and combined system in a stable state under different decision-making schemes.
\end{abstract}

\section{Introduction}

Supply chain management has always been a concern of enterprises. To help enterprises better implement effective supply chains, Pittiglio, Rabin, Todd \& McGrath (PRTM) and AMR Research (AMR) led the establishment of the Supply Chain Council (SCC) in 1996 and released supply chain operations reference (SCOR) model, realized the transformation from function-based management to process-based management, and improved the performance of the supply chain. With the emergence of cloud computing, Internet of things (IoT), artificial intelligence (AI), and other new information technologies (IT), supply chain management has a new background and requirements. New technologies can change the way of communication among the supply chain members. Before the 1960s and 1970s, due to technical limitations, the rapid flow and sharing of information cannot be achieved. In the early traditional SC model, each node enterprise is responsible for its inventory control, production, or distribution ordering activities, and each echelon only has its immediate customer information [1]. The application of new information technology makes it possible to easily share the main information on the supply chain (SC) nodes.

Gradually, some new supply chain models are formed and applied in practice. Among them, the more commonly used are the vendor-managed inventory (VMI) model and the third-party logistics management inventory (TMI) model [2] so that the problems faced by the traditional SC have undergone some changes. For example, the bullwhip effect can be improved. The bullwhip effect is a description of the image of demand information distorted in the SC [3].

The development of science and technology has promoted the vertical integration of the supply chain, and the 
management concept has also changed. More and more decision makers have magnified the decision perspective, and supply chain management presents the trend of vertical integration. More and more decisions are made based on local alliances and the whole supply chain. Also, there are many different types of uncertainties in the SC, such as changes in market demand, limited production capacity, the delay of transportation time, and so on [4]. Even, the level of decision makers also has a huge impact on the SC. All of the above factors will lead to the supply chain system (SCS) in an unstable state, which will increase the difficulty and cost of management. So, it makes great sense to study the complex dynamic behavior of the SC closely related to these factors from different perspectives.

\section{Literature Review}

The research on the dynamic behavior of supply chain systems started in the early 1960s. It first appeared in the classic work "Industrial Dynamics" by Forrester, which is the simplest manifestation of the dynamic complexity of supply chain systems [5] and was later named "bullwhip effect." Since then, the research literature on the dynamic behavior of the supply chain keeps emerging. These studies can be divided into two parts, including the dynamic behavior analysis of the linear system model and dynamic behavior analysis of the nonlinear system model.

In the early days of the concept of dynamic behavior emergence, many research studies were based on the inventory and order based production control system (IOBPCS) and analyzed as a linear system. Towill [6] analyzed a reasonable industrial dynamics model of an inventory control system, which is IOBPCS, by using transfer function techniques. A general rule for parameter settings which can then be used for "local" tuning in a large-scale industrial dynamics simulation is proposed. Disney et al. [7] outlined a method of developing a fitness measure for use in a genetic algorithm for assessing the performance of a generic production control system. Disney et al. [8] described a genetic algorithm for optimizing system performance, via five vectors including inventory recovery to "shock" demands, inbuilt filtering capability, robustness to production lead time variations, robustness to pipeline level information fidelity, and systems selectivity. In a vendor-managed inventory (VMI) supply chain, Disney and Towill [9] considered a well-established production and distribution scheduling algorithm termed automatic pipeline, inventory, and order based production control system (APIOBPCS). Simulation and analysis demonstrate that poor design can cause instability and the recommended parameter can avoid it. Dejonckheere et al. [10] introduced a general decision rule that avoids variance amplification and succeeds in generating smooth ordering patterns, even when the demand has to be forecasted. Lin and Wang [11] analyzed the stability of the system using the characteristic equation. It is proved that the intuitive operation of a supply system with demand forecasting will cause bullwhip and lead time alone would not cause bullwhip. Nagatani and Helbing [12] studied several feasible production strategies for stabilizing the supply chain in the linear supply chain. Disney [13] analyzed the quasiperiodicity, bullwhip effect, and stability of the supply chain inventory system. In these studies of a linear systems model, most assume that orders at all levels of the supply chain are satisfied, regardless of inventory constraints, mainly used to study and analyze the dynamic behavior of the supply chain, the bullwhip effect.

With the deepening of research, it is found that based on various assumptions, for example, orders at all levels of the supply chain are satisfied, regardless of inventory constraints, and the supply chain system is regarded as a simple linear system model. It leads to the inconsistency between the research results and the actual situation and fails to reveal the nonlinear phenomena except the bullwhip effect. Therefore, more and more research studies have been conducted to construct nonlinear system models to study the supply chain. Mosekilde and Laugesen [14] established a nonlinear inventory model under the nonnegative constraint of order quantity and found chaos in the system. Wang et al. [15] studied the stability of a constrained production and inventory system with a forbidden returns constraint. The results show that accurate lead time information is the key to eliminating inventory drift and instability, and ordering strategies must be reasonably designed according to actual lead times to avoid these fluctuations and differences. Garcia et al. [16] designed a supply chain switching control system to improve the stability of the supply chain through internal mode control technology. Wang et al. [17] assumed that the system input was a certain step demand model and return was forbidden, obtaining the stability, period, quasiperiod, and chaotic boundary of the system by solving the system characteristic values. Ma and Si [18] investigated the influence of delay and weight on the complex dynamic characteristics of the system. Si and $\mathrm{Ma}$ [19] established a triopoly output game model with multiple delays in the competition of green products. By analyzing the existence of equilibrium points and local asymptotic stability, the influence of parameters on system stability and complexity is studied. Zhan et al. [20] established the model of VMI-APIOBPCS in apparel supply chain and used the Routh-Hurwitz stability criterion to analyze the stability. Jin and Song [21] established a nonlinear system model to study the effects of two strategies of the nonlinear supply chain with and without a shortage on the dynamic behavior of the remanufacturing supply chain system. Lin and Naim [22] developed a hybrid ATO system dynamics model based on the well-established inventory and order based production control systems and analytically studied the impact of nonlinearities on its dynamic performance. $\mathrm{Xu}$ and Lee [23] presented A multiechelon supply chain system having parametric perturbations and disturbances to demonstrate chaotic nonlinear dynamical behaviors.

In the existing research on the complex dynamic behavior of the supply chain, many scholars used hypotheses to simplify the research objects and used the linear model to analyze the bullwhip effect in the supply chain. At the same time, many scholars consider practical factors such as prohibiting returns and limited supply capacity, constructing nonlinear dynamic models, and studying the 
impact of different demand scenarios, production models, and decision parameters on the stability of the supply chain, which has achieved rich results.

The analysis found that most studies can be divided into the category of local decision-making perspectives. However, in the existing research, no research mentions the decision-making perspective, nor does it study and analyze the complex behavior of the supply chain under different decision-making perspectives. In this paper, the idea of a decision perspective is introduced. Based on the local decision perspective and the global decision perspective, the difference equations and simulation models of the system are established, respectively, and the simulation experiments are carried out to compare and analyze the complex dynamic behavior of the supply chain inventory system from different perspectives. At the same time, the effect of safety stock parameters on the complex dynamic behavior of the supply chain is analyzed.

\section{Supply Chain System Dynamic Model}

3.1. System Description. The supply chain is based on a thirdparty logistics management inventory model (TMI) and includes a supplier, a retailer, and a third-party logistics service provider (3PLP). The operation process of the supply chain is shown in Figure 1. The inventory system of the supply chain includes three nodes: the production warehouse, the distribution center, and the retailer. The supplier purchases or produces its own raw materials and produces the raw materials as finished products. The retailer buys finished products from the supplier and sells them to end consumers. The supplier performs production according to the replenishment order issued by the warehouse to the production system. The warehouse is adjacent to the production workshop, and the finished products can be quickly delivered to the warehouse. Suppose the transportation cycle is 0 . In order to immediately respond to the retailer's ordering needs, build a distribution center near the retailer, assuming that the transportation cycle from the distribution center to the retailer is 0 . The inventory information of each node is transmitted to the decision system in real-time. The decision-making system will provide decision-making services for each node. When the inventory is lower than the safety stock, it will send a replenishment notification to the node, and then each node will send an order notification to the superior node. For the decision-making system, it can make decisions based on two decision-making perspectives. One is based on the perspective of local decision making, taking the production system and the warehouse-distribution system as a whole. The other is based on the perspective of global decision making, taking the entire supply chain as a whole. The local decision-making perspective does not include the retailer, regardless of the difference in the status of the node inventory goods and the goods in transit.

3.2. Model Parameters and Variables. To facilitate model description, the following relevant notations of model variables and parameters are introduced (Table 1).

\subsection{Systematic Difference Equations}

3.3.1. Demand Forecast. For the superior node of the supply chain, the demand forecast is based on the actual demand of terminal consumers. This forecasting method can reduce the amplification effect of orders in the supply chain, which is more reasonable than the demand forecasting based on the orders of subordinate nodes. The simple exponential smoothing method is often used in demand forecasting and has achieved good results. Therefore, this paper uses this method to forecast the demand. The prediction expression is shown in equation (1). In this study, two kinds of customer demand are considered, including random demand obeying normal distribution and random demand obeying uniform distribution. Assume that the mean of two kinds of customer demand is $\mu$.

$$
F(t)=\theta F(t-1)+(1-\theta) D(t),
$$

where $\theta$ is the exponential smoothing constant. Existing studies have shown that $0 \leq \theta<2$ is required for stability of the forecasting system. In the paper, let $\theta=0.5$.

3.3.2. Production Strategy. The study uses automated pipeline, inventory, and order based production control system (APIOBPCS), a commonly used method of production control. The specific meaning of APIOBPCS is that the order quantity (or production plan) is equal to the sum of the predicted demand quantity, the adjustment quantity to the actual inventory level, and the adjustment quantity to the inventory in transit (or work-in-process (WIP) inventory). Most of the existing literature on the complex behavior of the supply chain has adopted this strategy $[9,24]$.

Based on the local decision perspective of the supply chain, the actual inventory of the warehouse-distribution system is considered as the supplier's finished product inventory. The production strategy is expressed as follows:

$$
O_{1}(t)=\max \left(0, F(t)+\alpha_{S}\left(I_{1}^{0}-I_{1}(t)\right)+\alpha_{\mathrm{SL}}\left(Y^{0}-Y(t)\right)\right) .
$$

Based on the global decision perspective of the supply chain, the actual inventory of the entire supply chain is considered as the supplier's finished product inventory. The production strategy is expressed as follows:

$$
\mathrm{O}_{2}(t)=\max \left(0, F(t)+\alpha_{S}\left(I_{2}^{0}-I_{2}(t)\right)+\alpha_{\mathrm{SL}}\left(Y^{0}-Y(t)\right)\right) .
$$

3.3.3. Inventory Strategy. The supplier, the retailer, and the distribution center adopt periodic inventory strategy. The strategy is to replenish at regular intervals, each time to the target inventory level.

From the local decision perspective, the initial inventory $B_{1}(t)$ and the end-of-cycle inventory $I_{1}(t)$ of the warehousedistribution system are expressed as follows: 


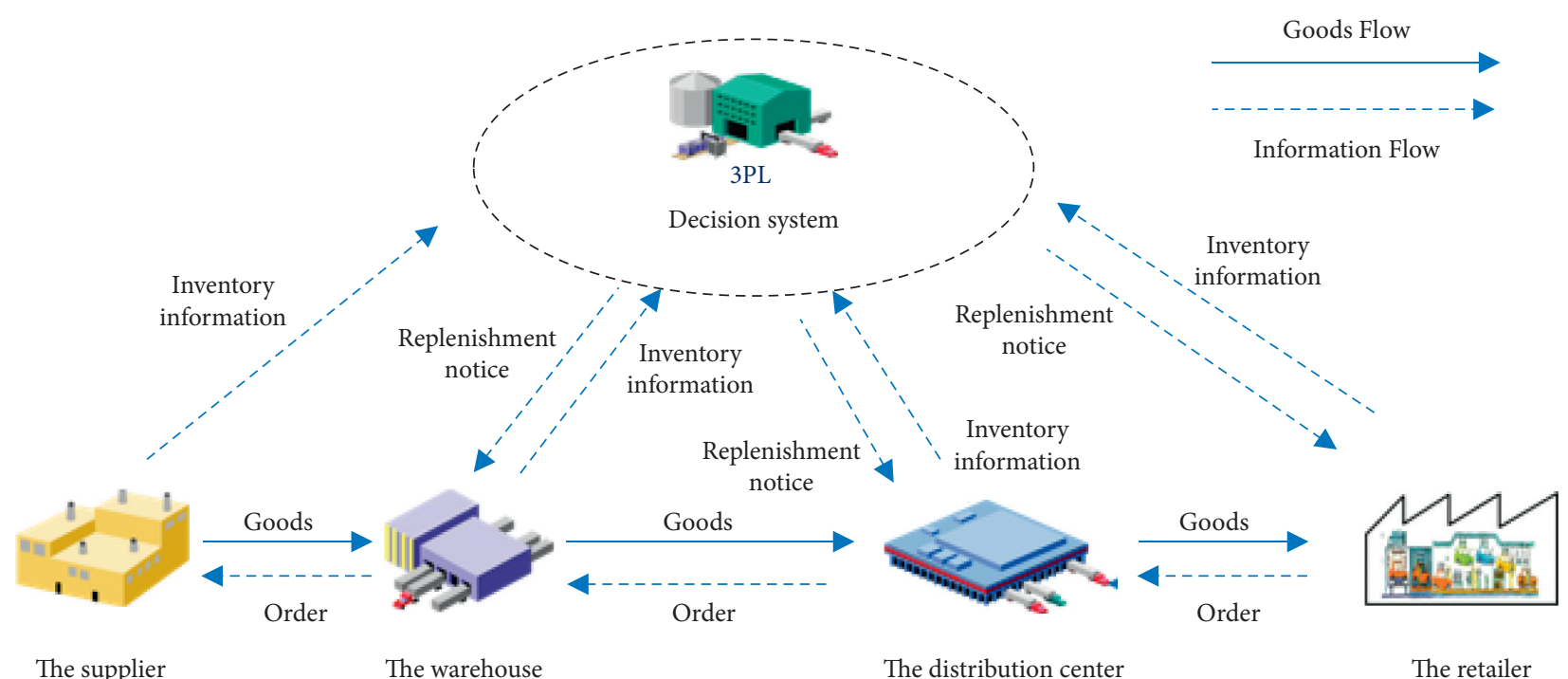

FIgUre 1: The TMI-SC operation flowchart.

TABLE 1: Model variables and parameters.

\begin{tabular}{|c|c|c|}
\hline \multicolumn{2}{|c|}{ Notation } & Description \\
\hline \multirow{21}{*}{ Variables } & $D(t)$ & The actual demand of consumers in period $t$ \\
\hline & $F(t)$ & The forecast demand in period $t$ \\
\hline & $I_{r}(t)$ & Retailer's initial inventory in period $t$ \\
\hline & $B_{r}(t)$ & Retailer's end-of-cycle inventory in period $t$ \\
\hline & $R_{r}(t)$ & Retailer's arrivals in period $t$ \\
\hline & $S_{r}(t)$ & Retailer's sales in period $t$ \\
\hline & $O_{r}(t)$ & Retailer's order quantity in period $t$ \\
\hline & $S_{d}(t)$ & Distribution center's shipments in period $t$ \\
\hline & $I_{d}(t)$ & Distribution center's initial inventory in period $t$ \\
\hline & $B_{d}(t)$ & Distribution center's end-of-cycle inventory in period $t$ \\
\hline & $R_{d}(t)$ & Distribution center arrivals in period $t$ \\
\hline & $W_{d}(t)$ & The in-transit inventory of the distribution center in period $t$ \\
\hline & $O_{d}^{a}(t)$ & The replenishment volume of the distribution center in period $t$ \\
\hline & $S_{w}(t)$ & Warehouse shipments in period $t$ \\
\hline & $I_{w}(t)$ & Warehouse's initial inventory in period $t$ \\
\hline & $B_{w}(t)$ & Warehouse's end-of-cycle inventory in period $t$ \\
\hline & $R_{w}(t)$ & Warehouse arrivals in period $t$ \\
\hline & $I_{i}(t)$ & $\begin{array}{c}\text { The initial inventory of system from the perspective of decision making in period } t \text {. When } i=1 \text {, it is local decision } \\
\text { perspective. When } i=2 \text {, it is global decision perspective. }\end{array}$ \\
\hline & $B(t)$ & The end-of-cycle inventory of system in period $t$ \\
\hline & $O_{i}(t)$ & $\begin{array}{l}\text { The replenishment of the system of system from the perspective of decision making in period } t \text {. When } i=1 \text {, it is local } \\
\text { decision perspective. When } i=2 \text {, it is global decision perspective. }\end{array}$ \\
\hline & $Y(t)$ & Supplier's WIP inventory in period $t$ \\
\hline \multirow{11}{*}{ Parameters } & $Y^{0}$ & The expected WIP inventory level of the supplier \\
\hline & $\alpha_{S}$ & The adjustment coefficient of inventory \\
\hline & $\alpha_{\mathrm{SL}}$ & The adjustment coefficient of WIP inventory \\
\hline & $T_{P}$ & Transport lead time \\
\hline & $T_{C}$ & Production lead time \\
\hline & $G_{r}$ & Safety inventory coefficient of the retailer \\
\hline & $G_{d}$ & Safety inventory coefficient of the distribution center \\
\hline & $G_{i}$ & $\begin{array}{l}\text { Safety inventory coefficient of the system from the perspective of decision making. When } i=1 \text {, it is local decision } \\
\text { perspective. When } i=2 \text {, it is global decision perspective. }\end{array}$ \\
\hline & $I_{r}^{0}$ & Retailer's expected inventory level \\
\hline & $I_{d}^{0}$ & Distribution center's expected inventory level \\
\hline & $I_{i}^{0}$ & $\begin{array}{c}\text { The expected inventory level of system from the perspective of decision making. When } i=1 \text {, it is local decision } \\
\text { perspective. When } i=2 \text {, it is global decision perspective. }\end{array}$ \\
\hline
\end{tabular}




$$
\begin{aligned}
B_{1}(t) & =B_{1}(t-1)-S_{d}(t-1)+R_{w}(t), \\
I_{1}(t) & =I_{1}(t-1)+R_{w}(t)-S_{d}(t), \\
I_{1}(t) & =B_{1}(t)-S_{d}(t), \\
I_{1}^{0} & =\mu G_{1} .
\end{aligned}
$$

From the global decision perspective, the inventory of the entire supply chain system is expressed as follows:

$$
\begin{aligned}
B_{2}(t) & =B_{2}(t-1)-S_{r}(t-1)+R_{w}(t), \\
I_{2}(t) & =I_{2}(t-1)+R_{w}(t)-S_{r}(t), \\
I_{2}(t) & =B_{2}(t)-S_{r}(t), \\
I_{2}^{0} & =\mu G_{2} .
\end{aligned}
$$

Due to the production delay, the following formula can be obtained:

$$
R_{w}(t)=O\left(t-T_{C}-1\right) .
$$
by

The expression of the supplier's WIP inventory is given

$$
Y(t)=Y(t-1)+O(t-1)-R_{w}(t)
$$

The expression of the replenishment quantity of the distribution center is shown in the following equation:

$$
O_{d}(t)=\max \left(0, F(t)+\alpha_{S}\left(I_{d}^{0}-I_{d}(t)\right)+\alpha_{\mathrm{SL}}\left(W^{0}-W_{d}(t)\right)\right) \text {. }
$$

The expression of the distribution center's inventory is given by equations (9)-(11). Moreover, the distribution center's expected inventory level is obtained by equation (12).

$$
\begin{gathered}
B_{d}(t)=B_{d}(t-1)-S_{d}(t-1)+R_{d}(t), \\
I_{d}(t)=I_{d}(t-1)+R_{d}(t)-S_{d}(t), \\
I_{d}(t)=B_{d}(t)-S_{d}(t), \\
I_{d}^{0}=\mu G_{d} .
\end{gathered}
$$

Among them, the distribution center's shipments in period $t$ are given by

$$
S_{d}(t)= \begin{cases}I_{d}(t-1)+R_{d}(t), & I_{d}(t-1)+R_{d}(t) \leq O_{r}(t-1), \\ O_{r}(t-1), & I_{d}(t-1)+R_{d}(t)>O_{r}(t-1) .\end{cases}
$$

Due to transportation delay, the following formula can be obtained:

$$
R_{d}(t)=O_{d}\left(t-T_{P}-1\right)
$$

The retailer is prohibited from returning goods, so the retailer's order quantity is expressed as follows:

$$
O_{r}(t)=\max \left(0, I_{r}^{0}-I_{r}(t)\right) .
$$

The expression of the retailer's inventory is given by

$$
\begin{aligned}
B_{r}(t) & =B_{r}(t-1)-S_{r}(t-1)+R_{r}(t), \\
I_{r}(t) & =I_{r}(t-1)+R_{r}(t)-S_{r}(t), \\
I_{r}(t) & =B_{r}(t)-S_{r}(t), \\
I_{r}^{0}(t) & =\mu G_{r} .
\end{aligned}
$$

The replenishment of the retailer can be quickly obtained from the distribution center. That is, the replenishment notice is issued at the end of the period, and the replenishment can be received at the beginning of the next period. So, the following expression can be obtained:

$$
R_{r}(t)=\min \left(O_{r}(t-1), I_{d}(t-1)+R_{d}(t)\right) .
$$

At the same time, the retailer's sales and consumer demand satisfied the following formula:

$$
S_{r}(t)= \begin{cases}I_{r}(t-1)+R_{r}(t), & I_{r}(t-1)+R_{r}(t) \leq D(t), \\ D(t), & I_{d}(t-1)+R_{d}(t)>D(t) .\end{cases}
$$

\section{The Block Diagram from Different Perspectives}

Using the discrete system $Z$-transform theory, according to the difference equation in the previous chapter, the block diagram of each supply chain node can be drawn.

\subsection{The Block Diagram of the Supply Chain}

4.1.1. The Block Diagram of Supply Chain Nodes from the Local Decision Perspective. For retailers, the information input of the inventory system is customer demand, and the information output is order quantity. For the distribution center, the information input of the inventory system is customer demand, retailer's order quantity, and inventory in transit, and the output information is the replenishment quantity. The block diagrams of the retailer and the distribution center are shown in Figures 2 and 3, respectively.

Based on the perspective of local decision making, for the production-warehouse system, the production-warehouse system and the distribution center are regarded as a combined system, and retailer inventory is not taken into account. The block diagram of the production-warehouse system is shown in Figure 4.

4.1.2. The Block Diagram of Supply Chain Nodes from the Global Decision Perspective. Based on the perspective of global decision-making, for retailers and distribution centers, the operation process, information input, and output of the inventory system are unchanged, so the block diagram is the same as that based on the local decision-making perspective. For the production-inventory system, the inventory of the entire supply chain including the retailer's inventory is used as the basis for decision making, and the input information is customer demand, distribution center replenishment volume, distribution center inventory, and retailer inventory. The block 


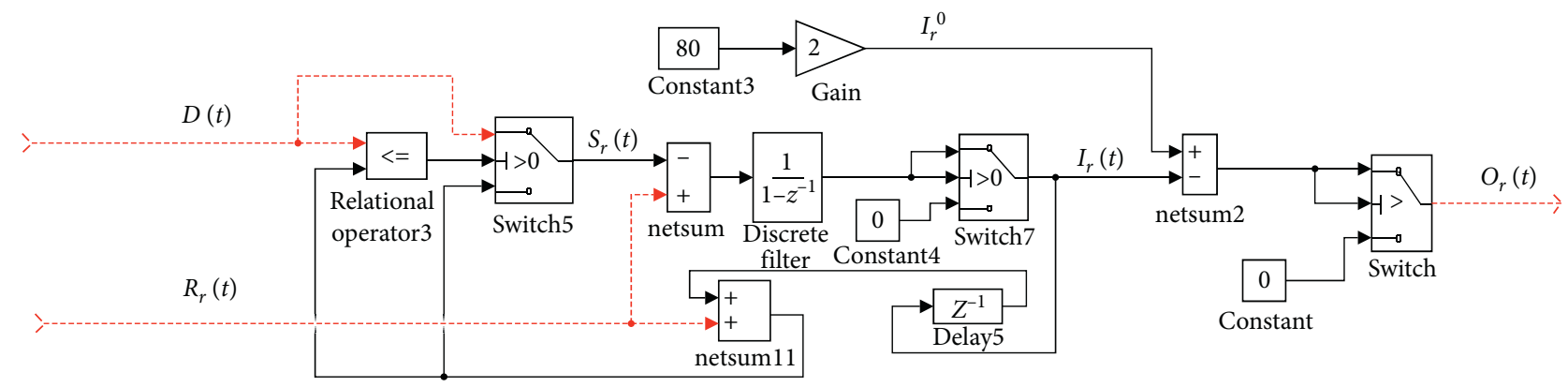

FIgURE 2: The block diagram of the retailer from the local decision perspective.

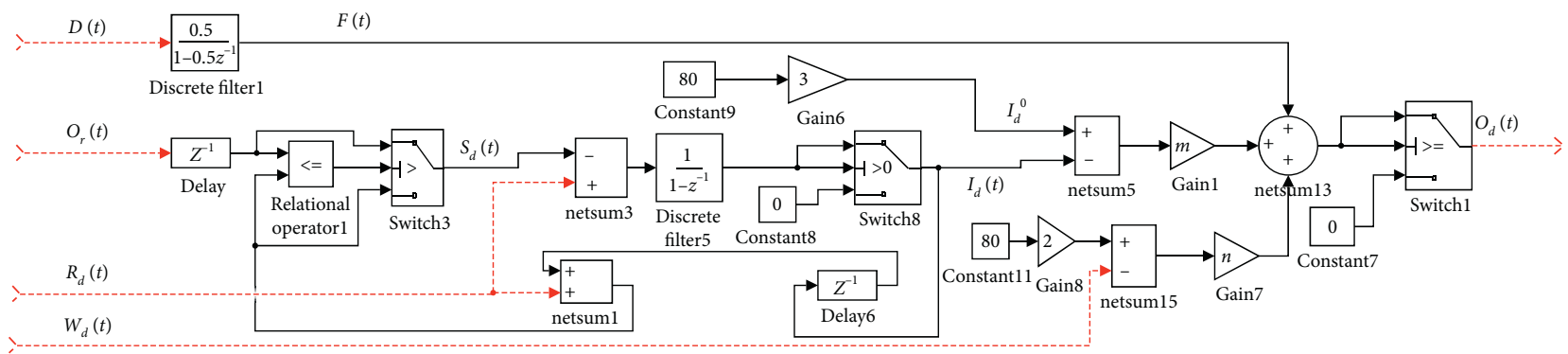

FIgURE 3: The block diagram of the distribution center from the local decision perspective.

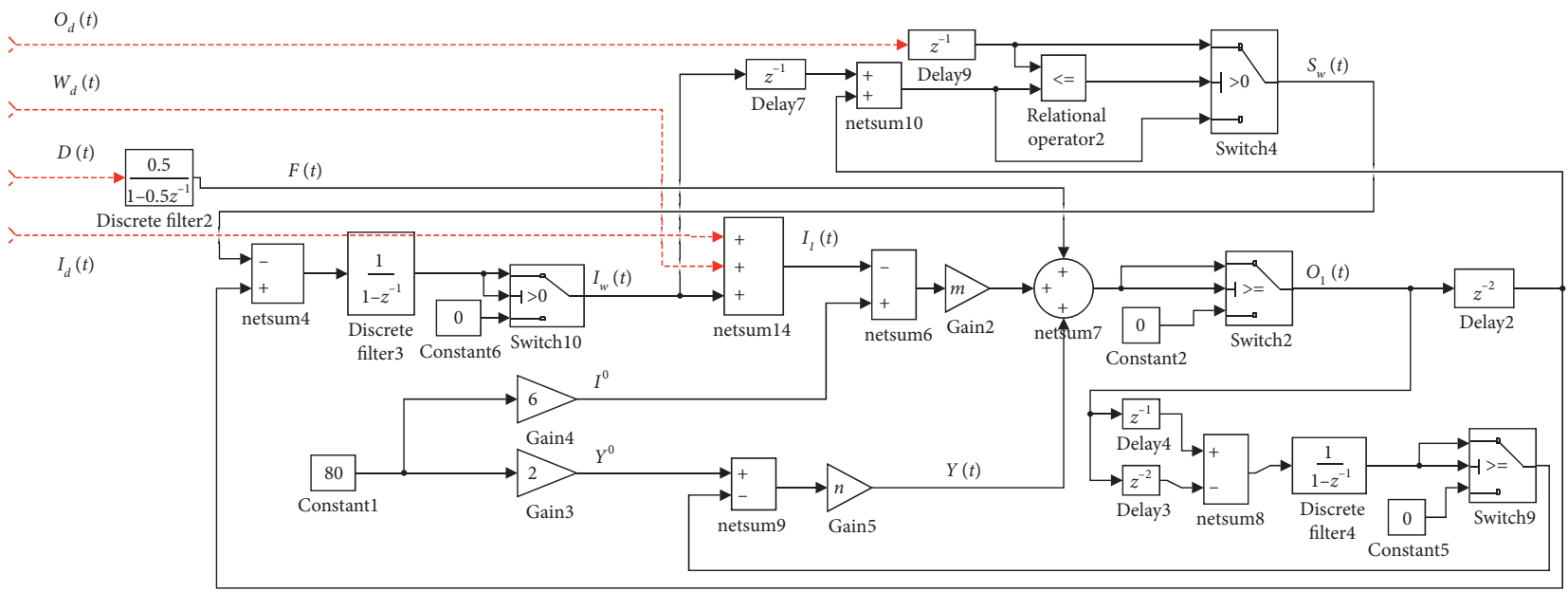

FIgURE 4: The block diagram of production and warehouse systems from the local decision perspective.

diagram of production and warehouse systems from the global decision perspective is shown in Figure 5.

4.2. The Block Diagram of the Whole Supply Chain. According to the models established above based on the local perspective and the global perspective, combined with the unitary transformation theory, the block diagrams of the system under the two models are shown in Figures 6 and 7.

\section{System Simulation and Data Analysis}

The largest Lyapunov exponent (LLE) is a standard to measure the stability of the system. Many studies use it to judge the stability of the system. When LLE is less than or equal to 0 , it indicates that the system is in a stable, periodic, or quasiperiodic state. It is an ideal state for ordering decisions. When LLE is greater than 0 , the system is in a chaotic or quasichaotic state. In the paper, the Wolf reconstruction method is used to calculate the largest Lyapunov exponent (LLE) value of each node or combined system to judge the stability of the system. The calculation principle of this method is as follows.

Suppose the time series is $X_{1}, X_{2}, \ldots, X_{n}$. Reconstruct the phase space and get $Y\left(t_{i}\right)=\left(X\left(t_{i}\right), X\left(t_{i+T}\right), \ldots, X\left(t_{i+(m-1) T}\right)\right)(i=1,2, \ldots, N)$, where $m$ is the embedding dimension and $T$ is the time delay. Take the initial point $Y\left(t_{0}\right)$, and let its distance from the nearest neighboring point $Y_{0}\left(t_{0}\right)$ be $L_{0}$. The time evolution of the two 


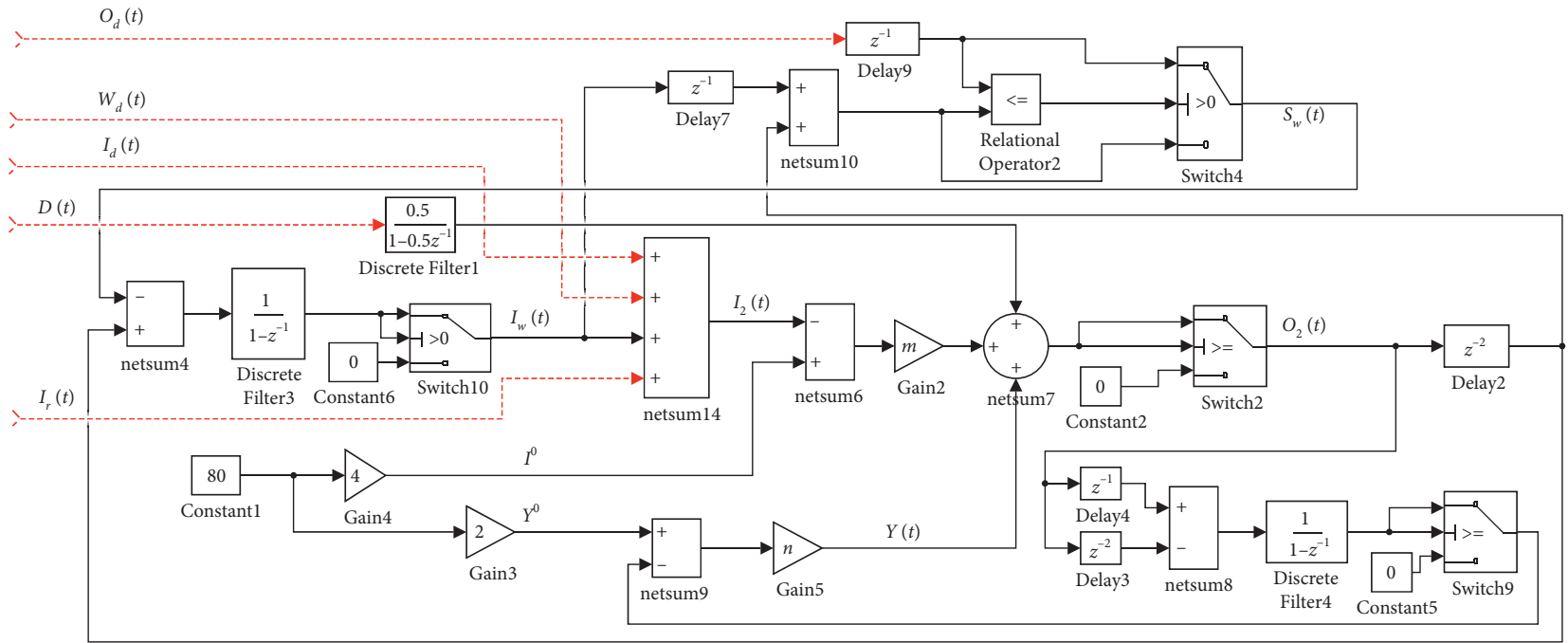

Figure 5: The block diagram of production and warehouse systems from the global decision perspective.

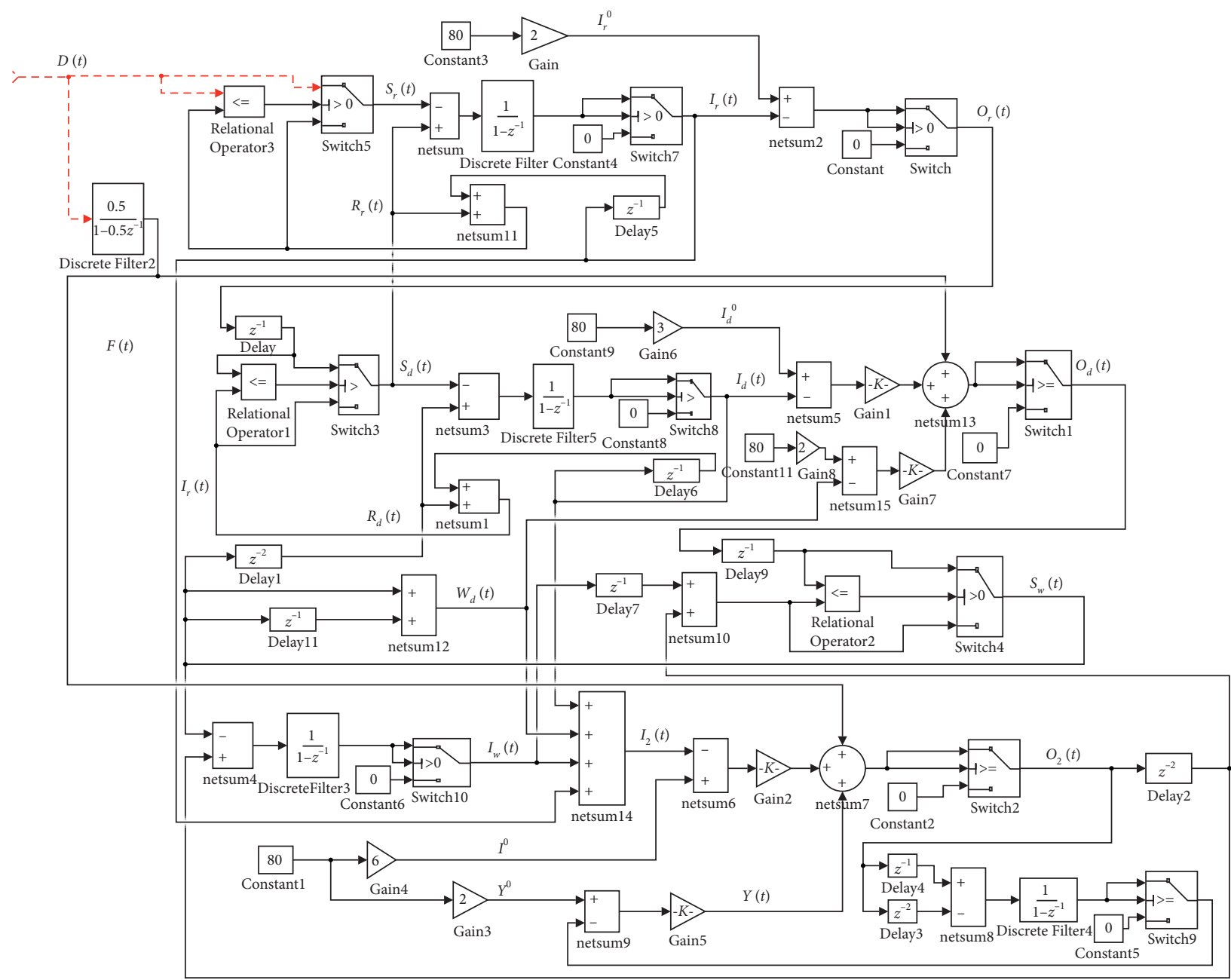

FIgURE 6: The block diagrams of the system under the global decision perspective.

points is traced until $t_{i}$, two points $Y\left(t_{1}\right)$ and $Y_{0}\left(t_{1}\right)$ are obtained, and the distance $L_{0}^{1}$ between them exceeds a specified value $\varepsilon$. Keep $Y\left(t_{1}\right)$ and find a point $Y_{1}\left(t_{1}\right)$ near it. The distance $L_{1}$ between the two points should be less than $\varepsilon$, and the angle formed should be as small as possible. The evolution process is continued until $Y(t)$ reaches the end $\mathrm{N}$ of the time 


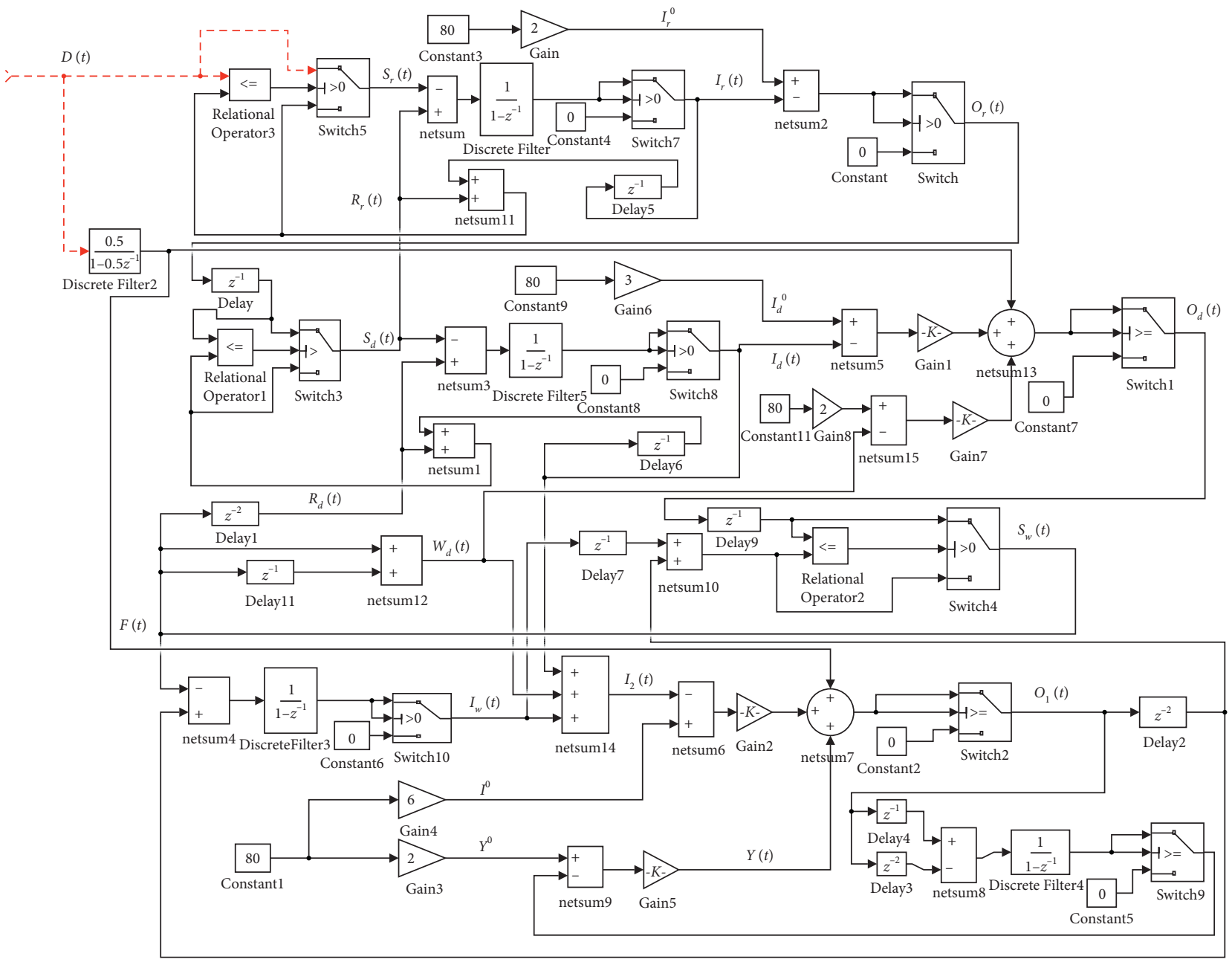

Figure 7: The block diagrams of the system under the local decision perspective.

series. The total number of iterations is $\mathrm{M}$. The formula for calculating the LLE is as follows:

$$
\lambda=\frac{1}{t_{M}-t_{0}} \sum_{i=0}^{M} \ln \frac{L_{i}^{1}}{L_{i}} .
$$

5.1. Decision Parameters. Based on the constructed model and block diagrams, we plan to further study the impact of different ordering strategies and inventory management strategies on the nonlinear supply chain system with restrictions on the prohibition of returns and limited inventory from different perspectives. Previous studies have shown that relevant order decision parameters such as inventory adjustment parameters have an important impact on the dynamic characteristics of the system.

We designed a simulation experiment under various order parameter combinations in the decision space $\left[\alpha_{S}, \alpha_{\mathrm{SL}}\right]$, and $\left[G_{d}, G_{i}\right]$. Then, using the simulation data, the LLE is calculated to analyze the impact of the different demand types, inventory adjustment coefficients, and safety inventory coefficients on system stability from different perspectives.

In general, the decision makers pay more attention to inventory adjustment, and the adjustment parameters are less than 1; this article assumes the value range of these two inventory adjustment coefficients is $0<\alpha_{\mathrm{SL}} \leq \alpha_{S}$ to $0.02 \leq \alpha_{S} \leq 1$. Both $\alpha_{S}$ and $\alpha_{\mathrm{SL}}$ have changed by 0.02 steps $[2,25]$. And three combinations of safety stock factors $\left[G_{d}, G_{i}\right]$ are selected, including $[1,4],[2,5]$, and $[3,6]$.

5.2. Simulation Analysis. According to the selection of the aforementioned decision parameter range, use Matlab to carry on the simulation experiment. Suppose that in this research supply chain, the production delay is 1 , the transportation delay is 2 , and the safety inventory coefficient of the retailer is 2 . That is, $T_{P}=2, T_{C}=1$, and $G_{r}=2$. At the same time, in order to ensure the effectiveness of the simulation experiment, the setting of the simulation period should not be too short. In this paper, the simulation period is set to 1000 . If the period is calculated in days, then it is the amount of data for nearly 3 years. In the actual problem, to analyze the company's inventory system, the three-year operation data are completely sufficient.

This article combines practical issues and considers two demand scenarios including the random demand that meets the normal distribution and the random demand that meets the uniform distribution. Both of these demand scenarios 
are more commonly used in real-world problems. The latter are more stringent and have higher requirements for supply chain response.

Firstly, based on a global perspective, under the scenario of random demand obeying normal distribution, we use the model shown in Figure 6 for simulation, calculate the index of each node of the supply chain according to the obtained data, and obtain the contour map under different combinations of safety stock factors, as shown in Figures 8-10.

By comparing and analyzing Figures $8-10$, we can find that, based on the global decision perspective, with the increase of the safety stock factor, the decision area in the stable state for each node of the supply chain and the system under the perspective gradually increases. As can be seen from Figure 8, based on the global perspective for decision making, when $G_{d}=1$ and $G_{1}=4$, under the scenario of random demand obeying normal distribution, the stability of the supply chain nodes under different inventory adjustment parameters is very different. Figure 8 (a) shows that the LLE value of the retailer's inventory system is less than 0 in the entire decision area of adjustment coefficients. However, Figure $8(\mathrm{~b})$ shows that the value of the LLE is greater than zero in most decision areas. Figure 8(c) shows that in most areas, the LLE value of the whole supply chain inventory is less than zero. That is to say, based on the perspective of global decision making, when $G_{d}=1$ and $G_{1}=4$, under the scenario of random demand obeying normal distribution, the retailer inventory system can be in a stable state regardless of any value of adjustment parameters. Under the same conditions, the distribution center inventory system is in an unstable state in most decision-making areas. For the entire supply chain from the perspective of decision making, if the state of the item is not considered, only the quantity is considered, and the system is stable in most decision-making areas. The research on the system from the perspective of decision making is based on the total quantity of goods in the system, and the nodes where the goods are located in the system may be different, and the state may be different. So, there will be a phenomenon where the system is in a stable state and the nodes in it are in an unstable state. As can be seen from Figures 9 and 10, regardless of whether $G_{d}=2$ and $G_{1}=5$ or $G_{d}=3$ and $G_{1}=6$, for retailers, the values of LLE are all less than zero in the entire decision area.

In addition, based on the global decision perspective, under the setting of different demand scenarios and the combination of safety stock parameters, the LLE of each node and combination system under 1275 adjustment parameter combinations was calculated separately. It is found that, based on the perspective of global decision making, there are 374 adjustment parameter combinations that can keep each node and combination system of the supply chain in a stable state in the case of positive distribution demand and $G_{d}=1$ and $G_{1}=4$. For the other two settings of safety stock parameters, there are 746 and 1109 different adjustment parameter decision schemes that can make the supply chain local and overall stable. At the same time, based on the calculation of the LLE for the entire decision area, we can find the adjustment coefficients that can keep the inventory system of each node of the supply chain and the entire supply chain inventory system in a stable state and the average value is the smallest. When $G_{d}=1$ and $G_{1}=4, \alpha_{S}=$ 0.38 and $\alpha_{\mathrm{SL}}=0.3$. When $G_{d}=2$ and $G_{1}=5, \alpha_{S}=0.24$ and $\alpha_{\mathrm{SL}}=0.14$. When $G_{d}=3$ and $G_{1}=6, \alpha_{S}=0.16$ and $\alpha_{\mathrm{SL}}=0.14$. Under different combinations of safety stock parameters, the number and optimal combination of adjustment parameter combinations that can keep the supply chain nodes and the combined system in a stable state are shown in Table 2.

Then, we change the demand scenario and perform simulation under the scenario of random demand obeying uniform distribution to obtain the contour map of each node under different combinations of safety stock coefficients, as shown in Figures 11-13.

As can be seen from Figures 11-13, under the scenario of random demand obeying uniform distribution, as the safety stock factor increases, the area where the LLE value of the entire supply chain inventory system and the inventory system of each node of the supply chain is less than zero gradually increases. The order of the area with the LLE value less than zero is the retailer, the whole supply chain, and the distribution center under certain safety stock factors. And it can be seen that for the distribution center and the entire supply chain, the area where the LLE value is less than zero exhibits a band distribution, which is immediately below $\alpha_{S L}=\alpha_{S}$. For retailer, in almost all regions, the LLE value is less than zero. However, the part with the smallest LLE value exhibits a dot-like and linear distribution, as shown in the dark parts of Figures 11(a), 12(a), and 13(a).

In addition, among the 1,275 kinds of adjustment parameter combinations, for different safety stock parameters, the number of parameter combinations that can keep each node of the supply chain and the combined system in a stable state is 146 , 334, and 532 in order. Among these parameters, when $G_{d}=1$ and $G_{1}=4$, if $\alpha_{S}=0.18$ and $\alpha_{\mathrm{SL}}=0.06$, the LLE value of the inventory system of each node of the supply chain and the entire supply chain inventory system are less than zero and the average value is the smallest. Similarly, when $G_{d}=2$ and $G_{1}=5, \alpha_{S}=$ 0.32 and $\alpha_{\mathrm{SL}}=0.26$. When $G_{d}=3$ and $G_{1}=6, \alpha_{S}=0.24$ and $\alpha_{\mathrm{SL}}=0.22$. Under different combinations of safety stock parameters, the number and optimal combination of adjustment parameter combinations that can keep the supply chain nodes and the combined system in a stable state are shown in Table 3.

Based on the local perspective, under the scenario of random demand obeying normal distribution, using the model shown in Figure 7 for simulation, we can get the contour map of each supply chain node under different combinations of safety stock factors, as shown in Figures 14-16.

It can be seen from Figures 14-16 that based on a local perspective, under the scenario of random demand obeying normal distribution, as the safety stock factors increases, the area where the LLE value of the inventory system of the production-warehouse-distribution system and some node of the supply chain is less than zero gradually increases. Under certain safety stock factors, the regional distribution of the LLE value does not show obvious characteristics. Especially when $G_{d}=2$ and $G_{2}=5$ and $G_{d}=3$ and $G_{2}=6$, the distribution of the LLE value in the decision area shows 


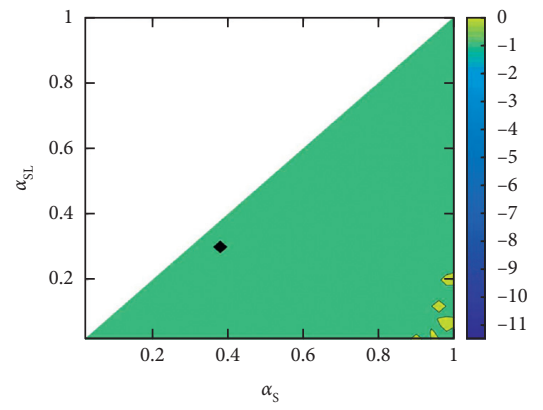

(a)

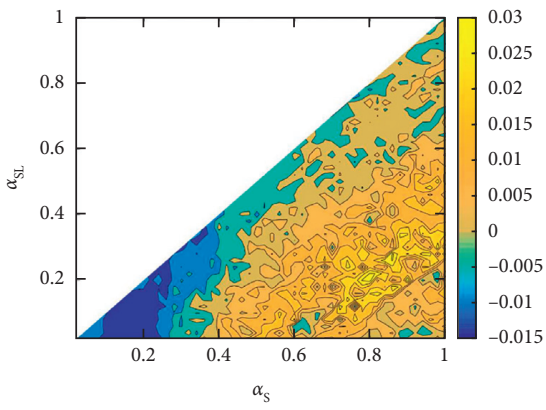

(b)

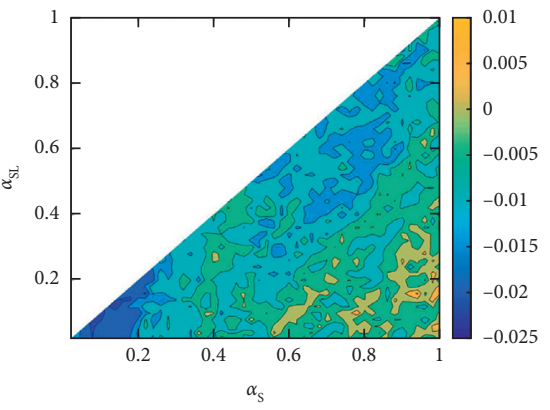

(c)

FIGURE 8: LLE diagram of supply chain nodes under the scenario of random demand obeying normal distribution from the global decision perspective when $G_{d}=1$ and $G_{2}=4$. (a) The retailer's LLE chart. (b) The LLE chart of the distribution center. (c) The LLE chart of the combined system from the decision perspective.

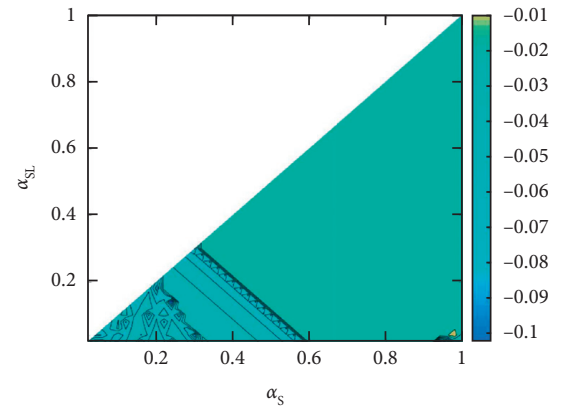

(a)

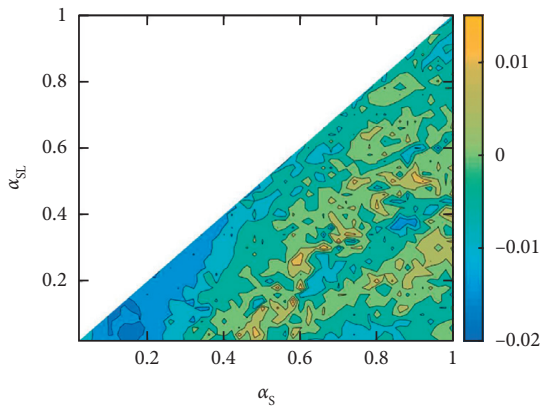

(b)

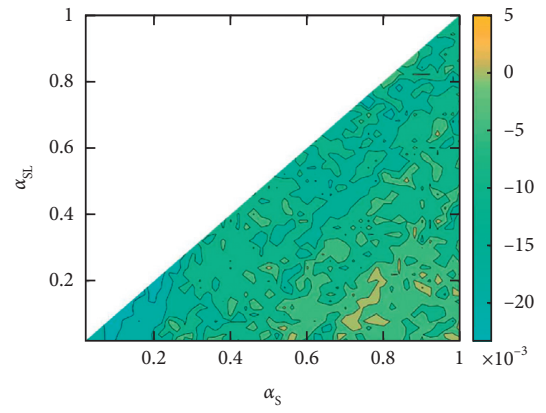

(c)

FigURE 9: LLE diagram of supply chain nodes under the scenario of random demand obeying normal distribution from the global perspective when $G_{d}=2$ and $G_{2}=5$. (a) The retailer's LLE chart. (b) The LLE chart of the distribution center. (c) The LLE chart of the combined system from the decision perspective.

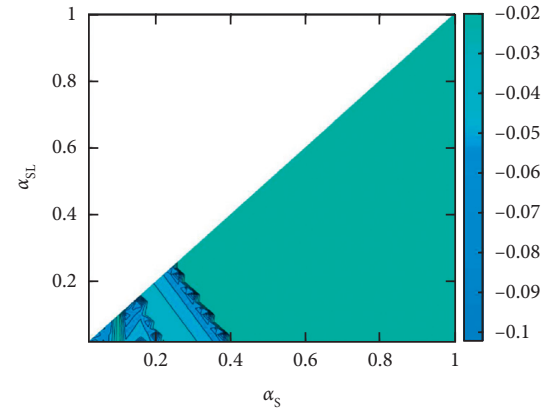

(a)

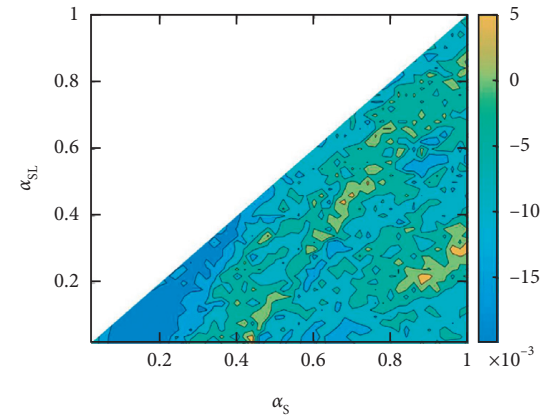

(b)

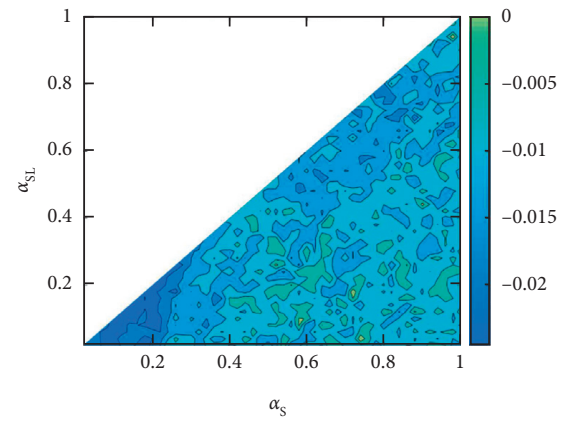

(c)

Figure 10: LLE diagram of supply chain nodes under the scenario of random demand obeying normal distribution from the global perspective when $G_{d}=3$ and $G_{2}=6$. (a) The retailer's LLE chart. (b) The LLE chart of the distribution center. (c) The LLE chart of the combined system from the decision perspective.

TABLE 2: The number and optimal combination of reasonable adjustment parameter combinations under the scenario of random demand obeying normal distribution from the global decision perspective.

\begin{tabular}{lcr}
\hline $\begin{array}{l}\text { Combination of safety } \\
\text { stock parameters }\left[G_{d}, G_{i}\right]\end{array}$ & $\begin{array}{c}\text { Number of reasonable adjustment } \\
\text { parameter combinations }\end{array}$ & Optimal combination $\left[\alpha_{S}, \alpha_{\mathrm{SL}}\right]$ \\
\hline$[1,4]$ & 374 & {$[0.38,0.3]$} \\
{$[2,5]$} & 746 & {$[0.24,0.14]$} \\
{$[3,6]$} & 1109 & {$[0.16,0.14]$} \\
\hline
\end{tabular}




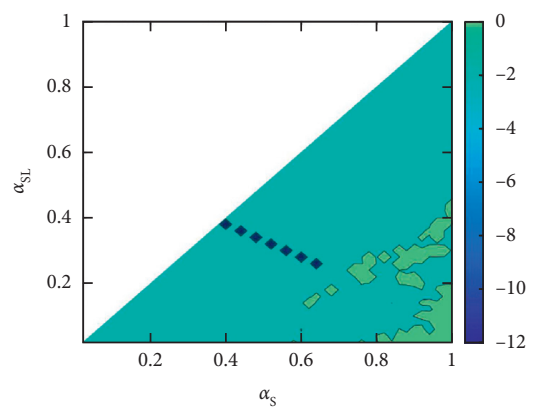

(a)

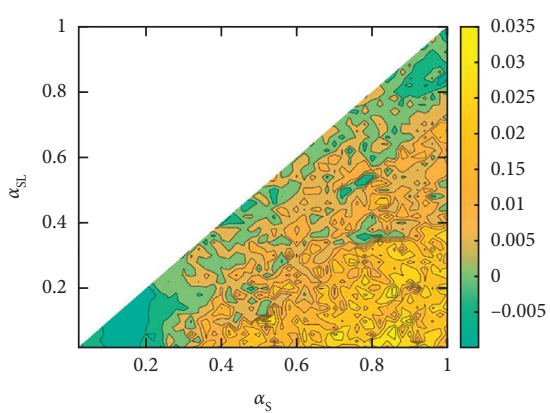

(b)

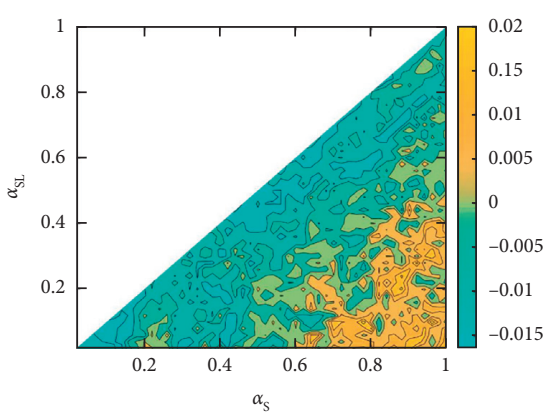

(c)

FIGURE 11: LLE diagram of supply chain nodes under the scenario of random demand obeying uniform distribution from the global perspective when $G_{d}=1$ and $G_{2}=4$. (a) The retailer's LLE chart. (b) The LLE chart of the distribution center. (c) The LLE chart of the combined system from the decision perspective.

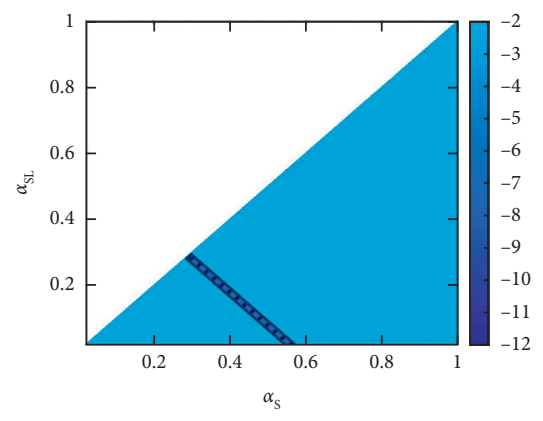

(a)

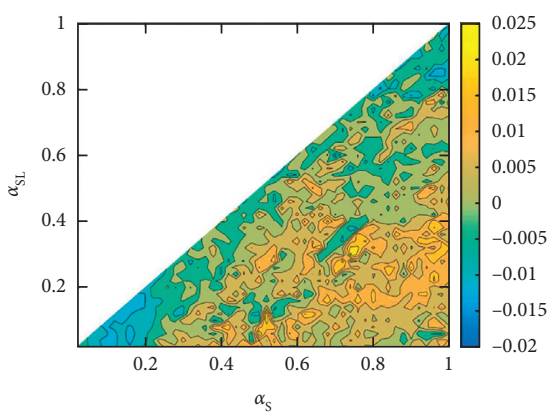

(b)

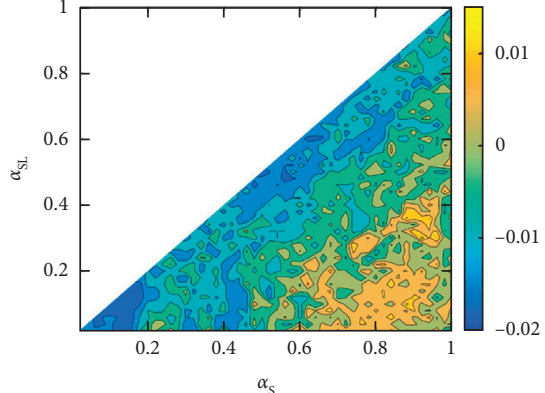

(c)

Figure 12: LLE diagram of supply chain nodes under the scenario of random demand obeying uniform distribution from the global perspective when $G_{d}=2$ and $G_{2}=5$. (a) The retailer's LLE chart. (b) The LLE chart of the distribution center. (c) The LLE chart of the combined system from the decision perspective.

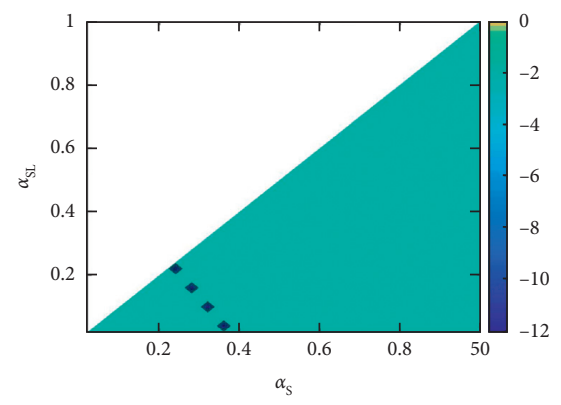

(a)

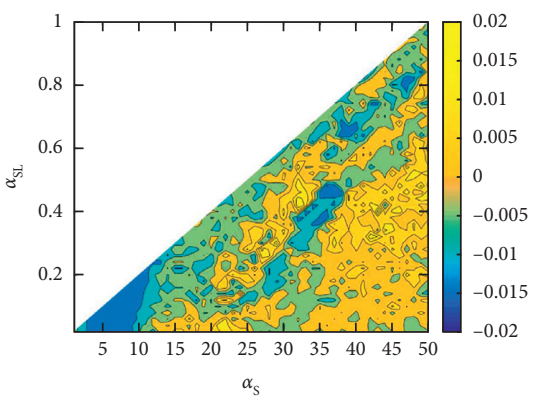

(b)

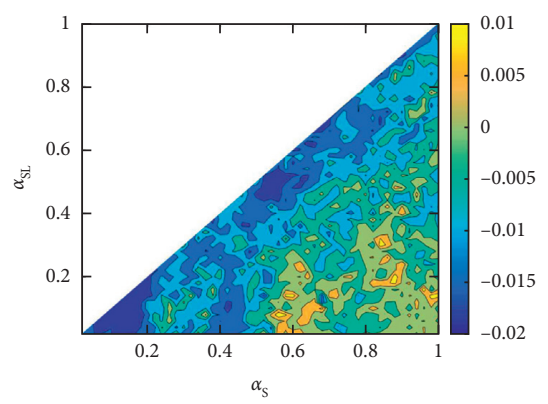

(c)

FIGURE 13: LLE diagram of supply chain nodes under the scenario of random demand obeying uniform distribution from the global perspective when $G_{d}=3$ and $G_{2}=6$. (a) The retailer's LLE chart. (b) The LLE chart of the distribution center. (c) The LLE chart of the combined system from the decision perspective.

an irregular state. From Figure 14, it can be seen that from the local perspective, when $G_{d}=1$ and $G_{2}=4$, for the retailer and the production-warehouse systems, the distribution of the larger part of the LLE value is relatively similar. The LLE value of distribution center is greater than zero in most decision-making areas. However, as can be seen from Figure 15, when $G_{d}=2$ and $G_{2}=5$, there are decision parameters that can keep the retailer's inventory system in an unstable state.
In addition, based on the local decision perspective, under the scenario of random demand obeying normal distribution, for different safety stock parameters, there are different numbers of reasonable decision-making schemes in the entire decision-making area. When $G_{d}=1$ and $G_{2}=4$, there are 380 reasonable adjustment parameter combinations. When $G_{d}=2$ and $G_{2}=5$, there are 775 reasonable adjustment parameter combinations. When $G_{d}=3$ and $G_{2}=6$, there are 1156 reasonable adjustment parameter 
TABLE 3: The number and optimal combination of reasonable adjustment parameter combinations under the scenario of random demand obeying uniform distribution from the global perspective.

\begin{tabular}{lcr}
\hline $\begin{array}{l}\text { Combination of safety } \\
\text { stock parameters }\left[G_{d}, G_{i}\right]\end{array}$ & $\begin{array}{c}\text { Number of reasonable adjustment } \\
\text { parameter combinations }\end{array}$ & Optimal combination $\left[\alpha_{S}, \alpha_{\mathrm{SL}}\right]$ \\
\hline$[1,4]$ & 146 & {$[0.18,0.06]$} \\
{$[2,5]$} & 334 & {$[0.32,0.26]$} \\
{$[3,6]$} & 532 & {$[0.24,0.22]$} \\
\hline
\end{tabular}

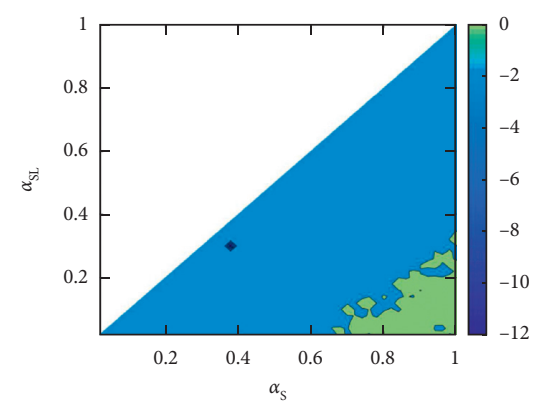

(a)

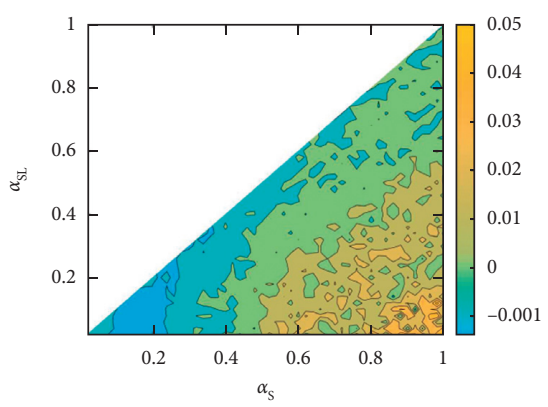

(b)

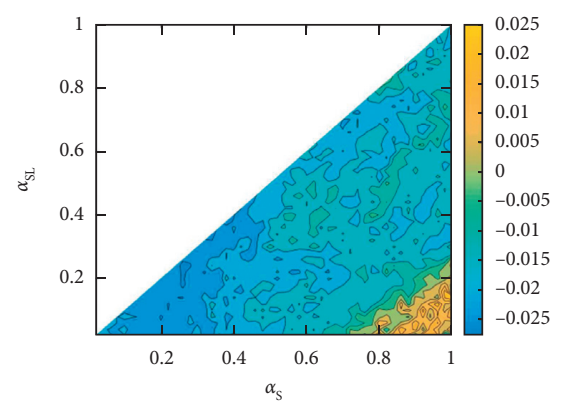

(c)

FIGURE 14: LLE diagram of supply chain nodes under the scenario of random demand obeying normal distribution from local perspective when $G_{d}=1$ and $G_{1}=4$. (a) The retailer's LLE chart. (b) The LLE chart of the distribution center. (c) The LLE chart of the combined system from the decision perspective.

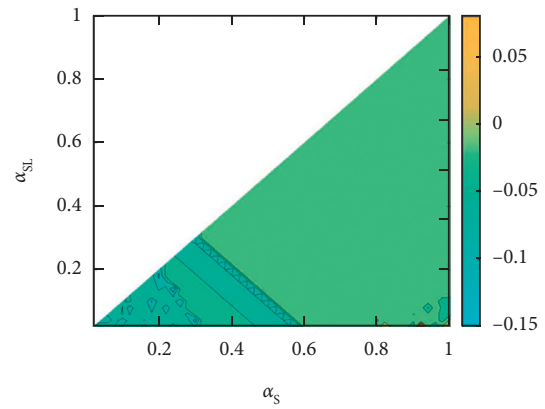

(a)

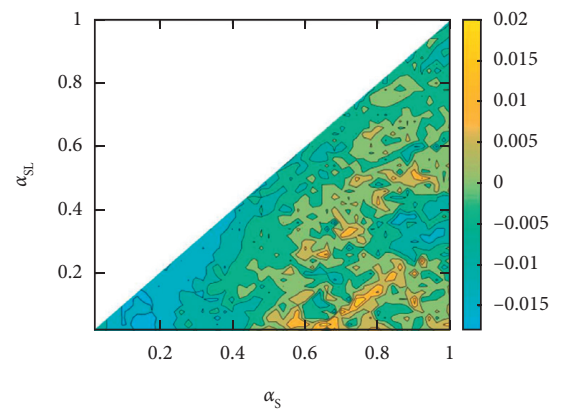

(b)

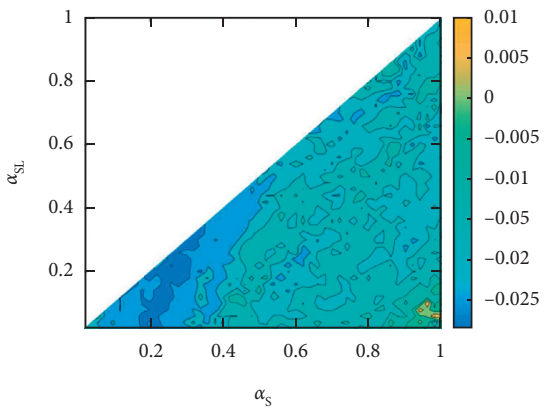

(c)

FIGURE 15: LLE diagram of supply chain nodes under the scenario of random demand obeying normal distribution from local perspective when $G_{d}=2$ and $G_{1}=5$. (a) The retailer's LLE chart. (b) The LLE chart of the distribution center. (c) The LLE chart of the combined system from the decision perspective.

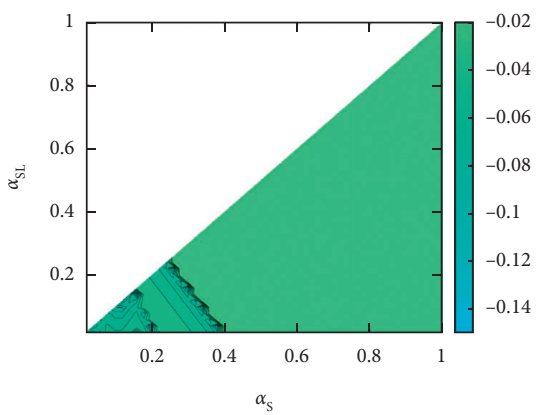

(a)

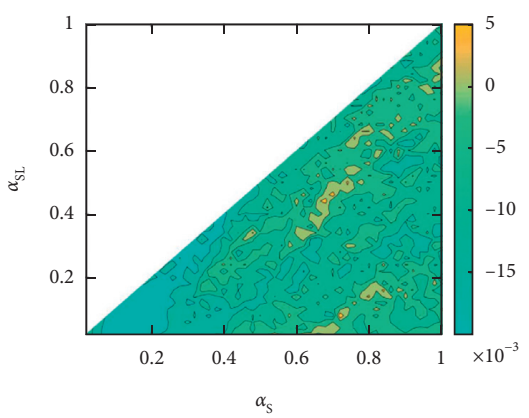

(b)

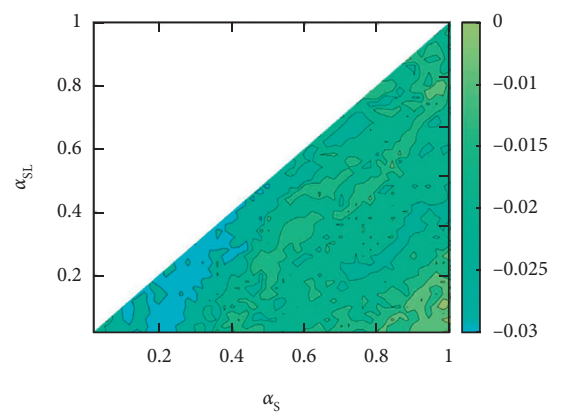

(c)

FIGURE 16: LLE diagram of supply chain nodes under the scenario of random demand obeying normal distribution from local perspective when $G_{d}=3$ and $G_{1}=6$. (a) The retailer's LLE chart. (b) The LLE chart of the distribution center. (c) The LLE chart of the combined system from the decision perspective. 
combinations. Among these parameter combinations that can stabilize the system, when $G_{d}=1$ and $G_{2}=4$, if $\alpha_{S}=$ 0.38 and $\alpha_{\mathrm{SL}}=0.3$, the LLE value of the inventory system of the production-warehouse-distribution system and each node of the supply chain are less than zero and the average value is the smallest. Similarly, when $G_{d}=2$ and $G_{2}=5, \alpha_{S}=$ 0.18 and $\alpha_{\mathrm{SL}}=0.06$. When $G_{d}=3$ and $G_{2}=6, \alpha_{S}=0.16$ and $\alpha_{\mathrm{SL}}=0.14$. Under different combinations of safety stock parameters, the number and optimal combination of adjustment parameter combinations that can keep the supply chain nodes and the combined system in a stable state are shown in Table 4.

Then, based on the local perspective, we change the demand scenario and perform simulation under the scenario of random demand obeying uniform distribution to obtain the contour map of each node under different combinations of safety stock coefficients, as shown in Figures 17-19.

As can be seen from Figures 17-19, under the scenario of random demand obeying uniform distribution, as the safety stock factor increases, the area where the LLE value of the inventory system of the production-warehouse-distribution system and each node of the supply chain is less than zero gradually increases. From Figure 17, it can be seen that for retailers, when $G_{d}=1$ and $G_{2}=4$, the smallest part of the LLE value presents a point-line distribution. In the same decision area, no other nodes present this feature. It can be seen from Figures 18 and 19 that as the safety stock parameter increases, for retailers, the area distribution of the smaller part of the LLE value shifts to the lower left in the entire decision area.

In addition, for the three combinations of safety stock parameters considered, there are 174, 361, and 542 reasonable adjustment parameter combinations that can keep the supply chain nodes and combination system in a stable state. In these reasonable decisions, when $G_{d}=1$ and $G_{2}=4$, if $\alpha_{S}=0.2$ and $\alpha_{S L}=0.12$, the LLE value of the inventory system of the production-warehouse-distribution system and each node of the supply chain are less than zero and the average value is the smallest. Similarly, when $G_{d}=2$ and $G_{1}=5, \alpha_{S}=0.16$ and $\alpha_{\mathrm{SL}}=0.14$. When $G_{d}=3$ and $G_{1}=6$, $\alpha_{S}=0.24$ and $\alpha_{\mathrm{SL}}=0.22$. Under different combinations of safety stock parameters, the number and optimal combination of adjustment parameter combinations that can keep the supply chain nodes and the combined system in a stable state are shown in Table 5.

According to the above analysis, it can be found that, on the whole, regardless of the local decision perspective or the global decision perspective, as the safety stock factors increase, the stability of each node of the supply chain gradually increases, and the requirements for adjustment parameters gradually decrease, that is, more adjusting parameters can keep the supply chain inventory system in a stable state. Regardless of the safety stock factors and the decision perspective, in the entire supply chain, the distribution center has the highest requirements for adjustment parameters, that is, the decision area of adjustment parameter where the distribution center is unstable is the largest. At the same time, based on the perspective of global decision making, a lower overall inventory of the supply chain can also keep the nodes in the supply chain and the
TABLE 4: The number and optimal combination of reasonable adjustment parameter combinations under the scenario of random demand obeying normal distribution from local perspective.

\begin{tabular}{lcc}
\hline $\begin{array}{l}\text { Combination of } \\
\text { safety stock } \\
\text { parameters }\left[G_{d}, G_{i}\right]\end{array}$ & $\begin{array}{c}\text { Number of reasonable } \\
\text { adjustment parameter } \\
\text { combinations }\end{array}$ & $\begin{array}{c}\text { Optimal } \\
\text { combination } \\
{\left[\alpha_{S}, \alpha_{\mathrm{SL}}\right]}\end{array}$ \\
\hline$[1,4]$ & 380 & {$[0.38,0.3]$} \\
{$[2,5]$} & 775 & {$[0.18,0.06]$} \\
{$[3,6]$} & 1156 & {$[0.16,0.14]$} \\
\hline
\end{tabular}

system under the perspective of a stable state. At the same time, an interesting phenomenon can be found. When only considering the quantity of goods, when the system composed of multiple nodes is in a stable state, the nodes in it may be in an unstable state. This is also a manifestation of the complexity of the system's dynamic behavior.

In order to more intuitively analyze the changes in inventory and the impact of demand types, adjustment parameters, and safety stock factors on the ability of each node of the supply chain and the system from different decision perspectives, a reasonable combination of adjustment parameters is selected for simulation experiments under different demand types and safety stock factors. This adjustment parameter combination can make the supply chain node and system in a stable state, and its average value is minimum.

In order to analyze the influence of different decisionmaking perspectives on the complex behavior of the supply chain, the inventory change chart of the nodes in the supply chain and the system under the perspective from the local perspective and the global perspective is drawn, as shown in Figures 20-25.

It can be seen from Figure 20 that under the scenario of random demand obeying normal distribution, when the retailer's inventory changes in a stable state, the performance of the retailer' inventory is basically the same under different decision-making perspectives. Inventory quickly increased from zero to safety stock level and then remained stable at 160 . This is a very good change feature, which verifies the effectiveness of the simulation model. At the same time, for different safety stock parameters, the retailer's stock changes are basically the same when they are in a stable state. As shown in Figure 21, under the scenario of random demand obeying uniform distribution, when it is stable, the retailer's inventory changes are basically the same as in the scenario of random demand obeying normal distribution. It shows that reasonable parameter settings can keep the supply chain in a stable state when the demand fluctuates greatly.

It can be seen from Figures 22 and 23 that under the scenario of random demand obeying normal distribution, when it is in a stable state, for the same safety stock factor, whether it is based on a local decision perspective or a global decision perspective, the distribution center inventory changes are basically the same. When $G_{d}=1$ and $G_{2}=4$, the distribution center inventory increases from zero to about 160 and then fluctuates around 160 . When $G_{d}=2$ and $G_{2}=5$, the inventory of the distribution center increases 


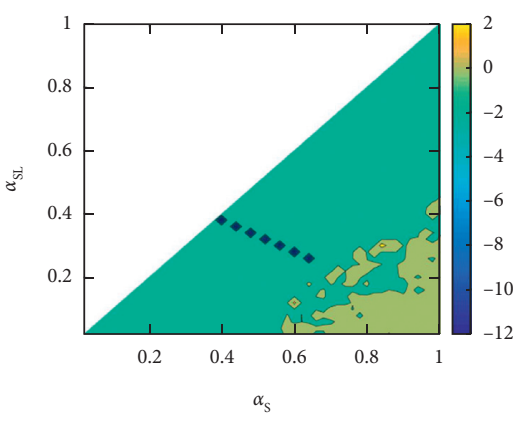

(a)

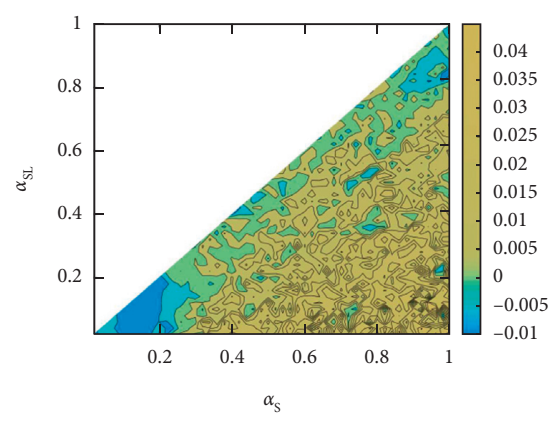

(b)

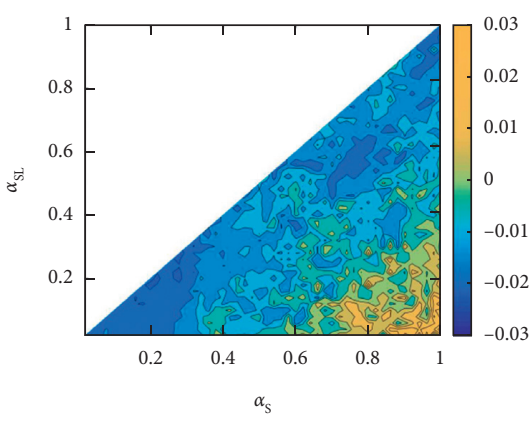

(c)

FIGURE 17: LLE diagram of supply chain nodes under the scenario of random demand obeying uniform distribution from local perspective when $G_{d}=1$ and $G_{1}=4$. (a) The retailer's LLE chart. (b) The LLE chart of the distribution center. (c) The LLE chart of the combined system from the decision perspective.

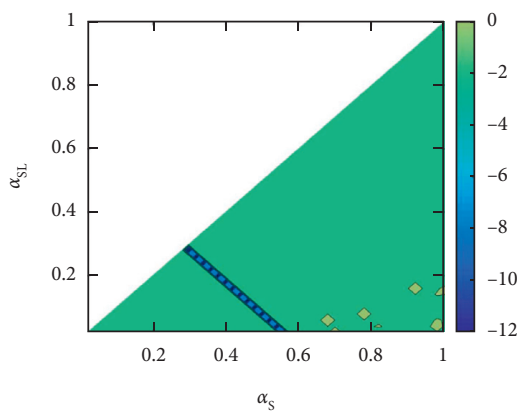

(a)

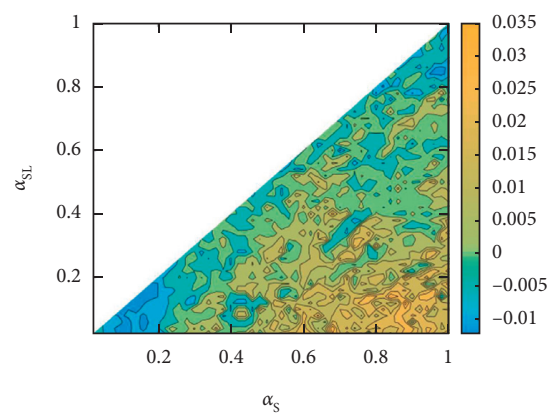

(b)

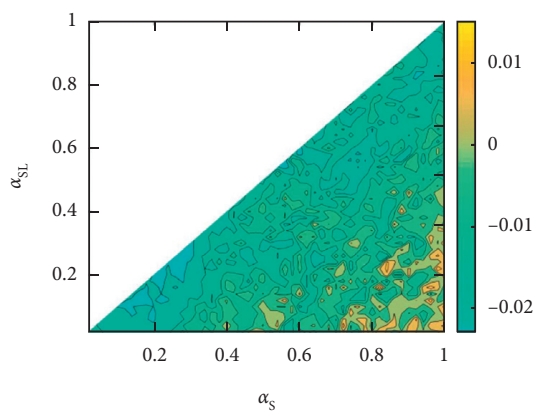

(c)

FIGURE 18: LLE diagram of supply chain nodes under the scenario of random demand obeying uniform distribution from local perspective when $G_{d}=2$ and $G_{1}=5$. (a) The retailer's LLE chart. (b) The LLE chart of the distribution center. (c) The LLE chart of the combined system from the decision perspective.

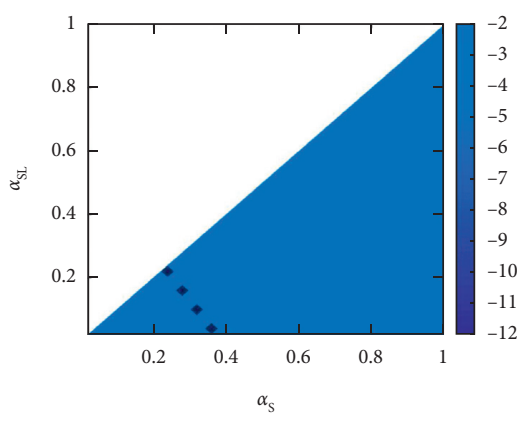

(a)

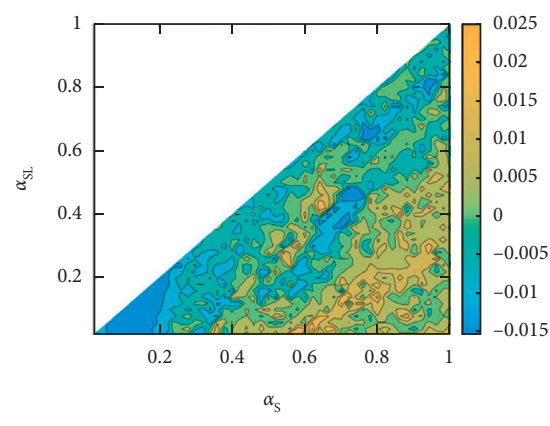

(b)

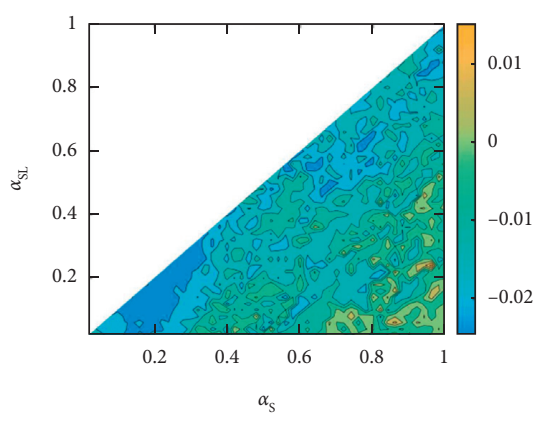

(c)

FIGURE 19: LLE diagram of supply chain nodes under the scenario of random demand obeying uniform distribution from local perspective when $G_{d}=3$ and $G_{1}=6$. (a) The retailer's LLE chart. (b) The LLE chart of the distribution center. (c) The LLE chart of the combined system from the decision perspective.

from zero to about 240 and then fluctuates around 240. When $G_{d}=3$ and $G_{2}=6$, the distribution center inventory increases from zero to about 320 and then fluctuates around 320. The fluctuation ranges under the three safety stock factor combinations are basically the same. As shown in Figure 23, under the scenario of random demand obeying uniform distribution, when in a stable state, the fluctuation range of the distribution center inventory change becomes significantly larger. It is verified that demand changes can
TABle 5: The number and optimal combination of reasonable adjustment parameter combinations under the scenario of random demand obeying uniform distribution from local perspective.

\begin{tabular}{lcc}
\hline $\begin{array}{l}\text { Combination of } \\
\text { safety stock } \\
\text { parameters }\left[G_{d}, G_{i}\right]\end{array}$ & $\begin{array}{c}\text { Number of reasonable } \\
\text { adjustment parameter } \\
\text { combinations }\end{array}$ & $\begin{array}{c}\text { Optimal } \\
\text { combination } \\
{\left[\alpha_{S}, \alpha_{\mathrm{SL}}\right]}\end{array}$ \\
\hline$[1,4]$ & 174 & {$[0.2,0.12]$} \\
{$[2,5]$} & 361 & {$[0.16,0.14]$} \\
{$[3,6]$} & 542 & {$[0.24,0.22]$} \\
\hline
\end{tabular}




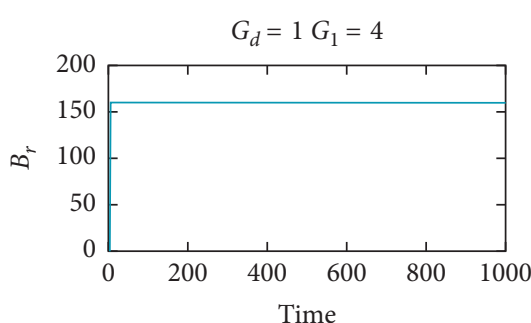

_ The global perspective

- The local perspective

(a)

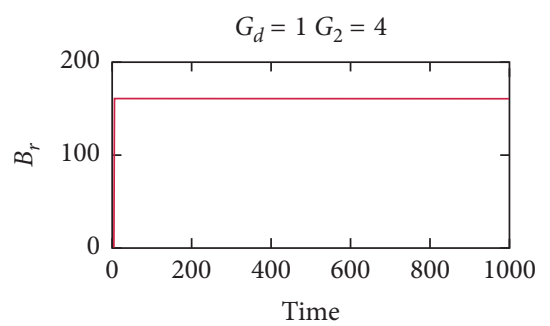

_ The global perspective

_ The local perspective

(d)

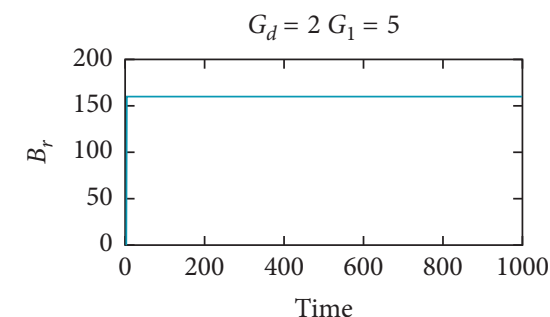

_ The global perspective

_ The local perspective

(b)

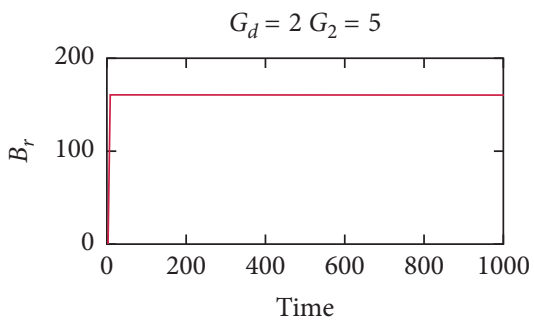

_ The global perspective

_ The local perspective

(e)

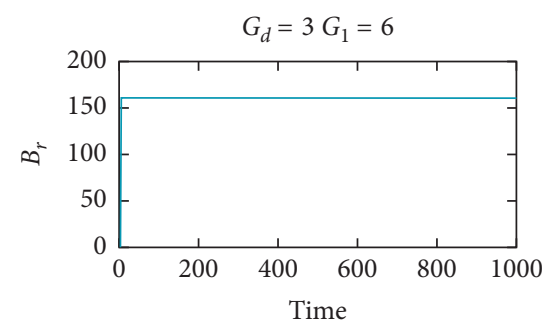

- The global perspective

_ The local perspective

(c)

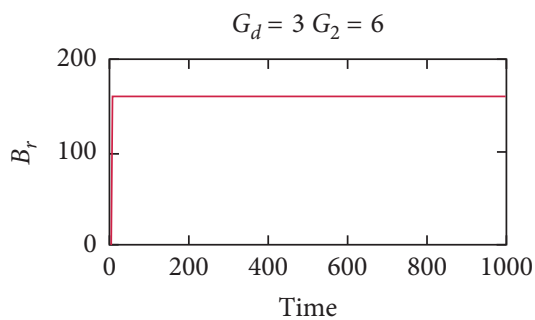

_ The global perspective

_ The local perspective

(f)

FIGURE 20: The initial inventory changes of the retailer in a stable state under the scenario of random demand obeying normal distribution from different perspectives.

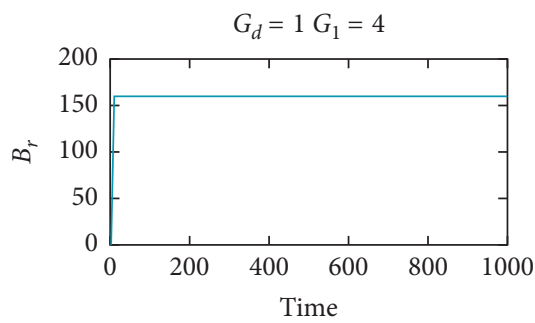

- The global perspective

_ The local perspective

(a)

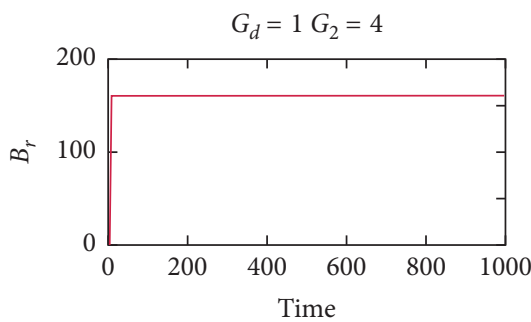

The global perspective

- The local perspective

(d)

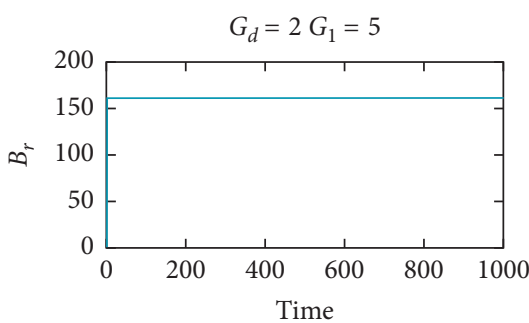

_ The global perspective

_ The local perspective

(b)

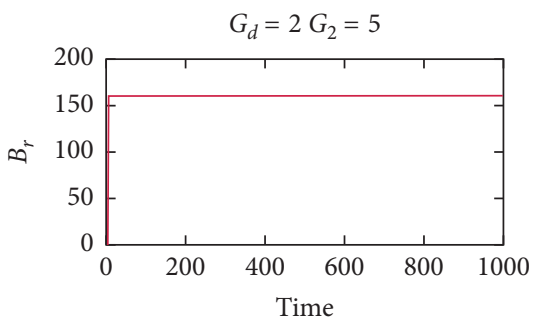

- The global perspective

_ The local perspective

(e)

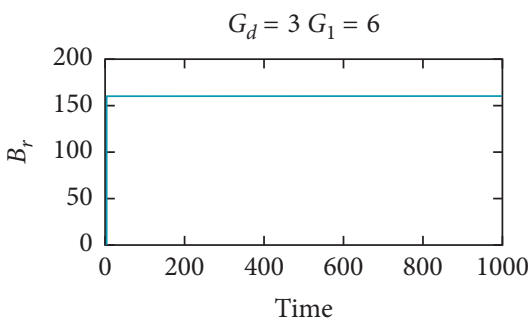

_ The global perspective

_ The local perspective

(c)

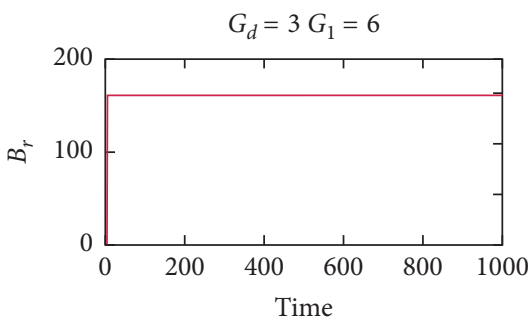

- The global perspective

_ The local perspective

(f)

Figure 21: The initial inventory changes of the retailer in a stable state under the scenario of random demand obeying uniform distribution from different perspectives. 


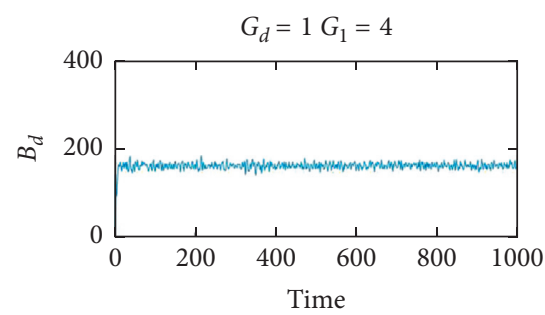

_ The global perspective

_ The local perspective

(a)

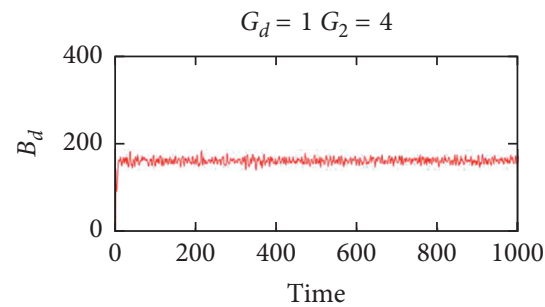

- The global perspective

_ The local perspective

(d)

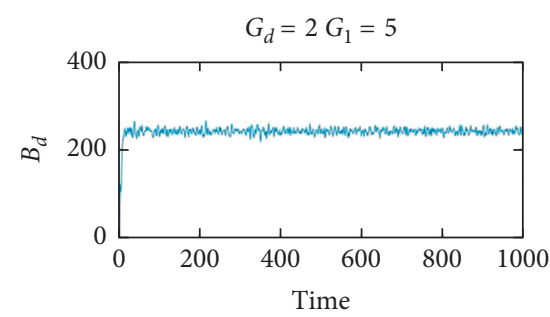

- The global perspective

— The local perspective

(b)

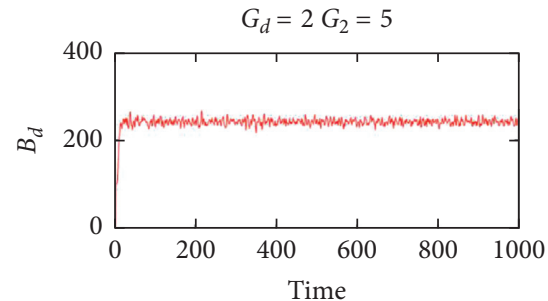

— The global perspective

_ The local perspective

(e)

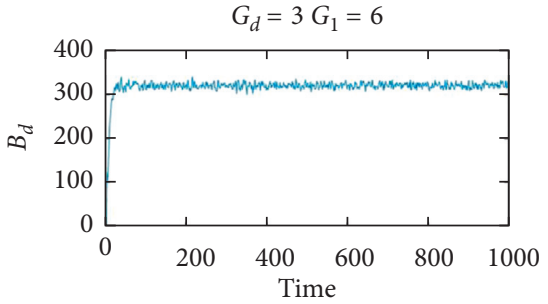

- The global perspective

_ The local perspective

(c)

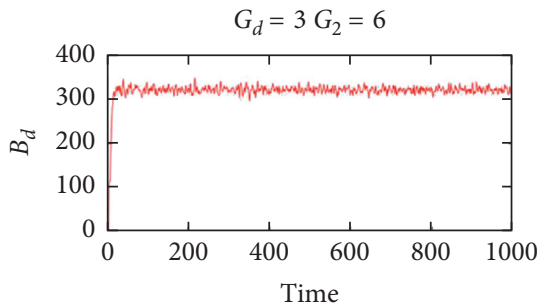

_ The global perspective

_ The local perspective

(f)

FIGURE 22: The initial inventory changes of the distribution center in a stable state under the scenario of random demand obeying normal distribution from different perspectives.

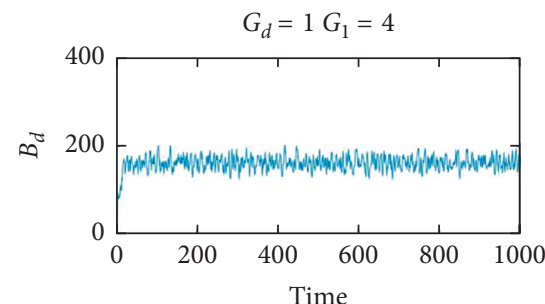

_ The global perspective

The local perspective

(a)

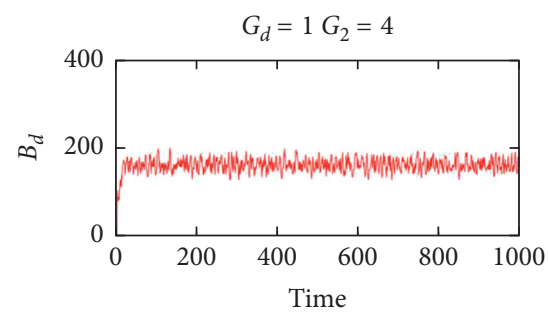

- The global perspective

- The local perspective

(d)

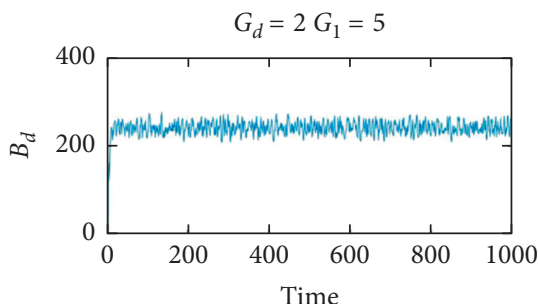

_ The global perspective

_ The local perspective

(b)

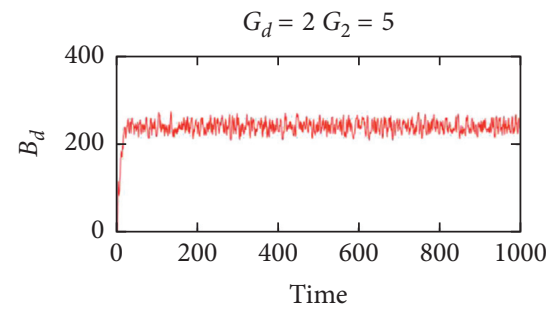

_ The global perspective

_ The local perspective

(e)

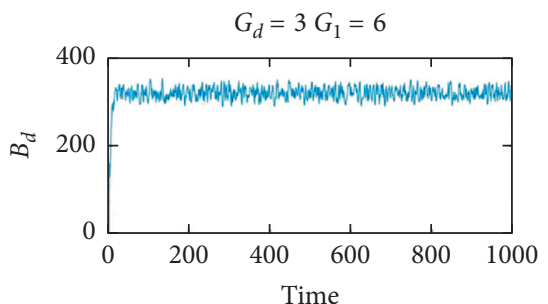

_ The global perspective

_ The local perspective

(c)

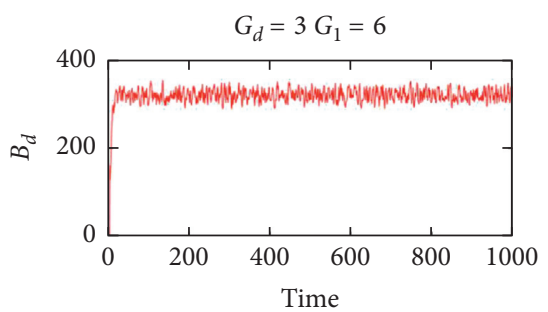

_ The global perspective

_ The local perspective

(f)

Figure 23: The initial inventory changes of the distribution center in a stable state under the scenario of random demand obeying uniform distribution from different perspectives. 


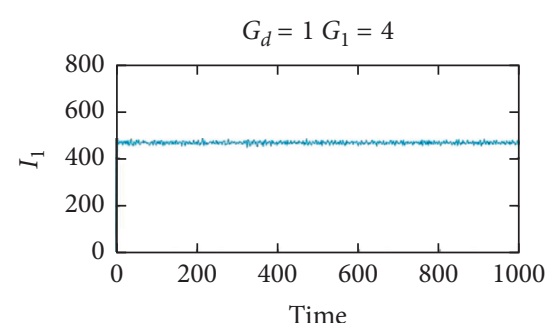

_ The global perspective

_ The local perspective

(a)

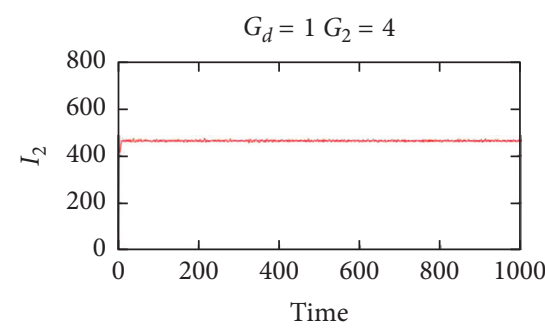

- The global perspective

_ The local perspective

(d)

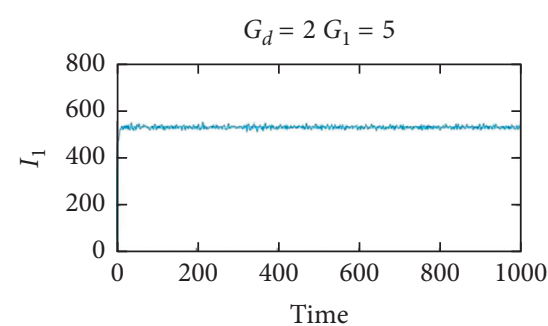

_ The global perspective

_ The local perspective

(b)

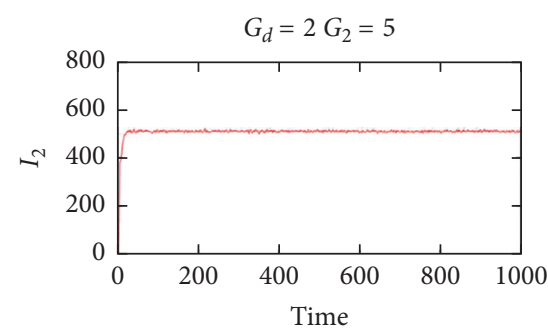

_ The global perspective

_ The local perspective

(e)

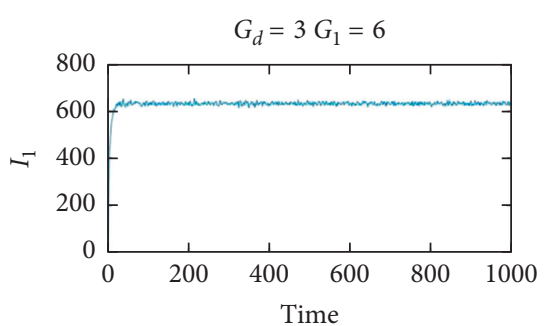

_ The global perspective

_ The local perspective

(c)

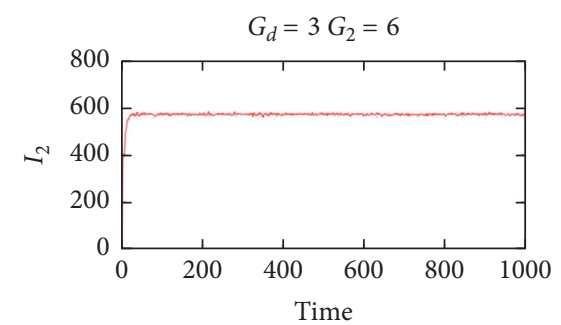

_ The global perspective

_ The local perspective

(f)

FIgURe 24: The initial inventory changes of the system from the perspective of decision under the scenario of random demand obeying normal distribution from different perspectives.

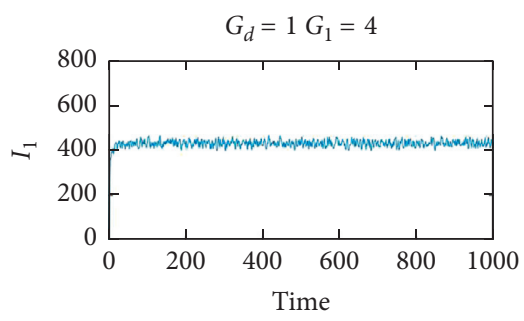

- The global perspective

- The local perspective

(a)

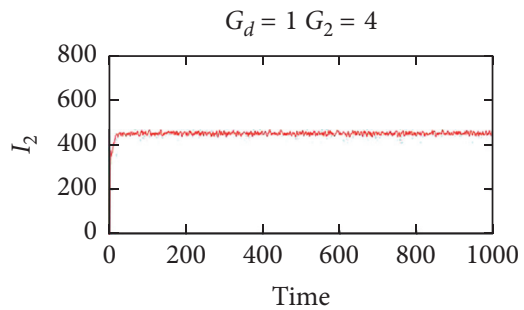

_ The global perspective

_ The local perspective

(d)

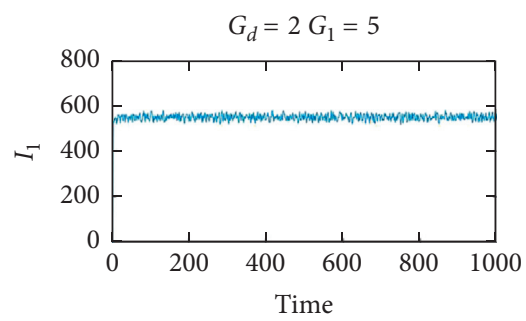

- The global perspective

_ The local perspective

(b)

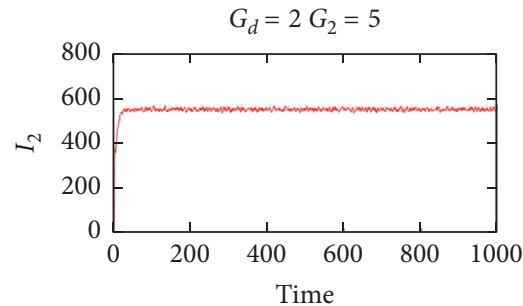

_ The global perspective

_ The local perspective

(e)

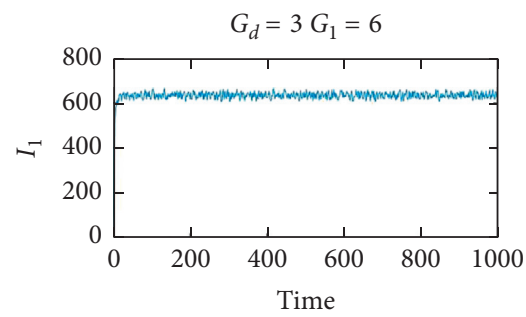

_ The global perspective

_ The local perspective

(c)

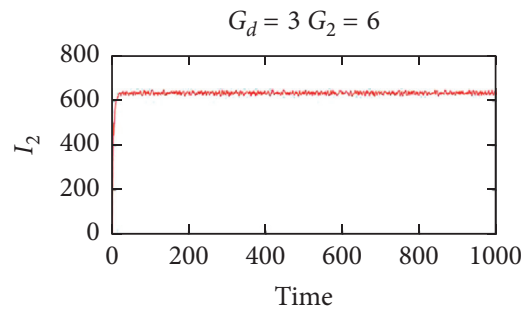

_ The global perspective

_ The local perspective

(f)

Figure 25: The initial inventory changes of the system from the perspective of decision under the scenario of random demand obeying uniform distribution from different perspectives. 


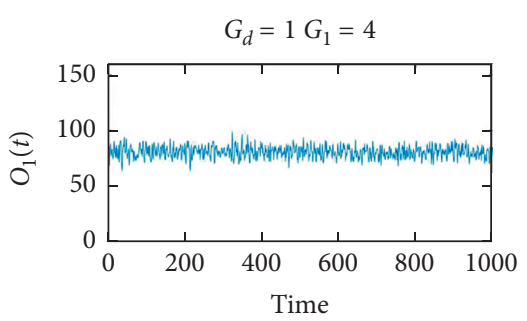

_ The global perspective

_ The local perspective

(a)

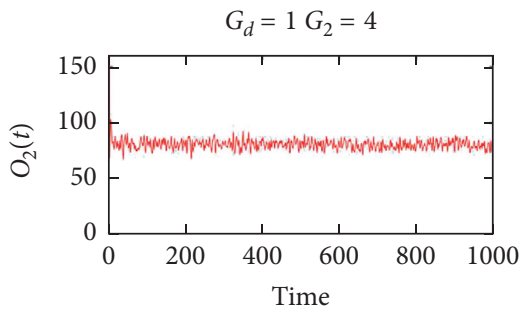

— The global perspective

_ The local perspective

(d)

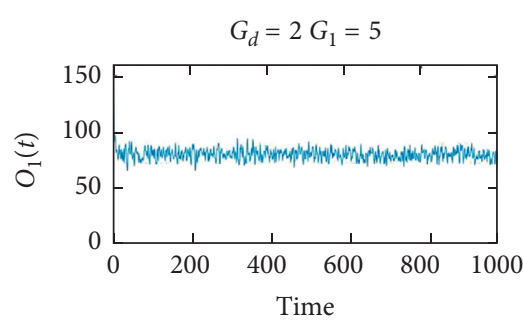

_ The global perspective

_ The local perspective

(b)

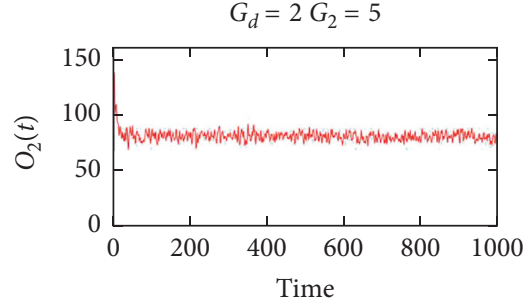

_ The global perspective

_ The local perspective

(e)

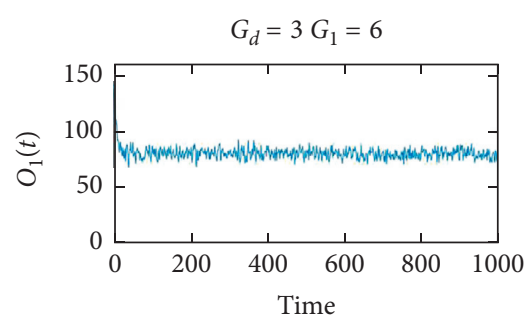

— The global perspective

_ The local perspective

(c)

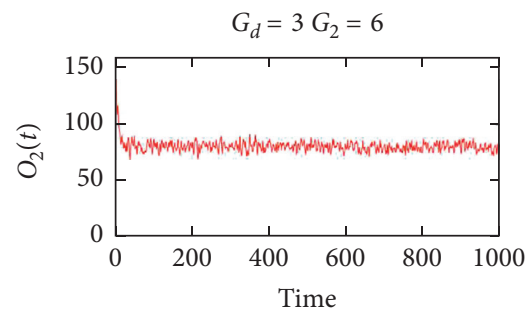

_ The global perspective

_ The local perspective

(f)

FIGURE 26: The production fluctuation under the scenario of random demand obeying normal distribution from different perspectives.

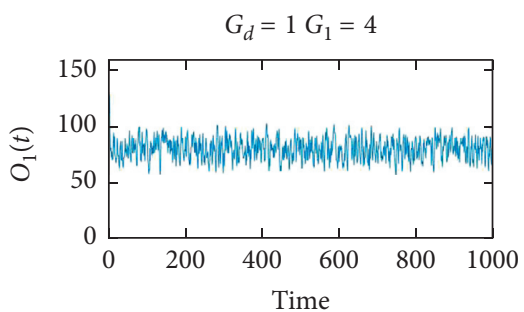

_ The global perspective

- The local perspective

(a)

$G_{d}=1 G_{2}=4$

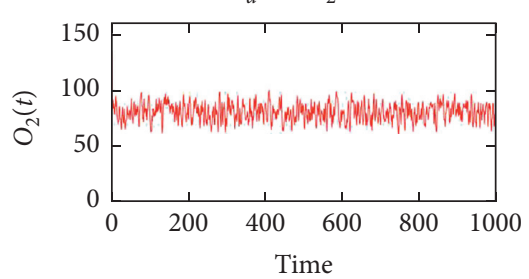

- The global perspective

- The local perspective

(d)

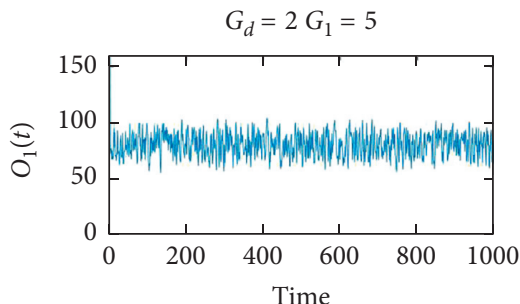

_ The global perspective

_ The local perspective

(b)

$G_{d}=2 G_{2}=5$

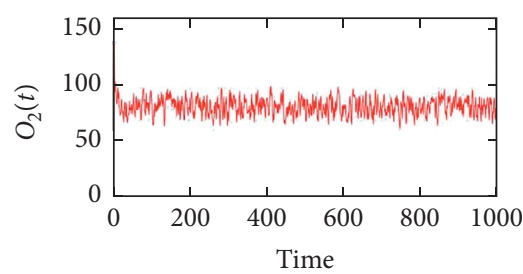

_ The global perspective

_ The local perspective

(e)

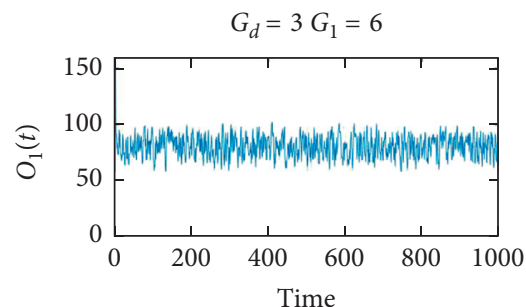

_ The global perspective

_ The local perspective

(c)

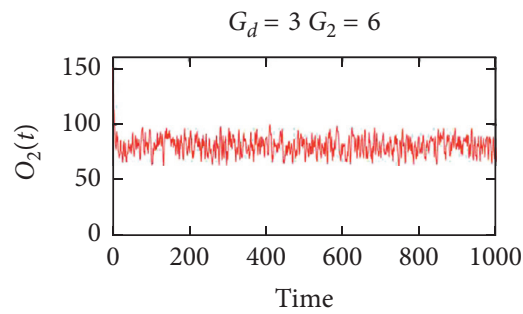

The global perspective
The local perspective

(f)

FIgURE 27: The production fluctuation under the scenario of random demand obeying uniform distribution from different perspectives. 
have an impact on the dynamic behavior of supply chain systems. However, the inventory levels are basically equal in the two demand scenarios.

For the combined system under the decision perspective, regardless of the state of the goods, as can be seen from Figures 24 and 25, under specific demand scenarios and safety stock parameters, the combined system under different decision perspectives has a very similar inventory change at steady state. At the same time, it was found that for the distribution center and the combined system, when it is in a stable state, the inventory level in the scenario of random demand obeying normal distribution is lower than the inventory level in the scenario of random demand obeying uniform distribution. As can be seen from Figure 24, under the scenario of random demand obeying normal distribution, when the safety stock parameter combination is $[1,4]$, based on the global decision perspective, when the supply chain system is in a stable state, the overall stock level is about 460 . Under the same demand scenario and safety stock parameter combination, based on the perspective of local decision making, the combined system inventory level under the perspective is 460 . In other words, the entire supply chain system does not include the retailer, and the inventory of other parts has reached 460. Similarly, when the safety stock parameter combination is $[2,5]$, the overall stock level is about 520 based on the global decision perspective. Based on the local decision perspective, the combined system inventory level under the perspective is about 505 . When the safety stock parameter combination is $[3,6]$, the overall stock level is about 627 based on the global decision perspective. Based on the local decision perspective, the combined system inventory level under the perspective is about 567.

Under the scenario of random demand obeying uniform distribution, when the supply chain system is in a stable state, Figure 25 shows the inventory level and fluctuation status under different safety stock parameter combinations and decision perspectives. When the safety stock parameter combination is $[1,4]$, the overall stock level is about 425 based on the global decision perspective. Based on the local decision-making perspective, the combined system inventory level under the perspective is 446 . When the safety stock parameter combination is $[2,5]$, the overall stock level is about 547 based on the global decision perspective. Based on the local decision perspective, the combined system inventory level under the perspective is about 505 . When the safety stock parameter combination is $[3,6]$, based on the global decision perspective, the overall stock level is about 632. Based on the local decision perspective, the inventory level of the combined system under the perspective is about 631. Therefore, for any combination of safety inventory parameters and any demand scenarios, based on a global decision perspective, choosing a reasonable adjustment parameter scheme can reduce the overall inventory level of the supply chain. The overall inventory volatility has increased slightly, but it does not affect the stability of the overall inventory.

In the supply chain, the stability of production has a great influence on the cost, and the production system produces according to the order quantity of the warehouse. In order to analyze the fluctuation of production in a stable state more intuitively, we draw the production fluctuation chart, as shown in Figures 26 and 27.

As can be seen from Figures 26 and 27, under steady conditions, the volatility of production appears to be stable at the desired level of demand, with slight fluctuations. Compared with demand, it is found that the volatility of production is basically the same as the fluctuation of demand, and the fluctuation of production is smaller than that of demand. It shows that the system can smoothen the change of demand very well.

\section{Conclusion}

Based on the needs of practical problems, this paper introduces the global decision-making thoughts in order to adapt to the trend of supply chain integration and puts forward different decision-making perspectives of supply chain inventory system management. Based on the proposed local decision perspective and global decision perspective, the complex dynamic behavior of the supply chain inventory system is studied. At the same time, the influence of the safety inventory setting on the dynamic behavior of the supply chain inventory system is studied.

The study found that, based on the perspective of global decision making, choosing a reasonable adjustment parameter scheme can reduce the overall inventory level of the supply chain. The overall inventory volatility has increased slightly, but it does not affect the stability of the overall inventory. When the safety stock parameter combination is $[1,4]$, based on the global decision perspective, the overall stock level is about 425 . Based on the local decision-making perspective, the combined system inventory level under the perspective is 446 . Based on a global decision perspective, the overall inventory can be reduced by about 180 . When the safety stock parameter combination is $[2,5]$, the overall stock can be reduced by about 118 . When the safety stock parameter combination is $[3,6]$, the overall stock can be reduced by about 159 . At the same time, based on different decision-making perspectives, the status distribution maps of each node or combination system under different demand scenarios and the most reasonable parameter settings are obtained.

On the whole, regardless of the local decision perspective or the global decision perspective, as the safety stock parameters increase, the stability of each node of the supply chain gradually increases, and the requirements for adjustment parameters gradually decrease. The analysis also found that each node has a different sensitivity to the adjustment parameters. Changes in adjustment parameters have little effect on the retailer. Under different scenarios and decision parameters, the retailer is in a stable state in almost the entire decision area. However, changes in adjustment parameters have a significant impact on the distribution center. In addition, the study also found that, whether based on a local decision perspective or a global decision perspective, the overall inventory level is in a stable 
state and maybe in an unstable state for internal nodes. This has important guiding significance for actual operation.

Although this paper selects three safety stock parameter combinations for research and obtains some effective conclusions, it does not study the entire safety stock parameter decision area. In the follow-up, we hope that interested scholars will conduct further research on the impact mechanism of safety stock parameters on the complex dynamic behavior of the supply chain.

\section{Data Availability}

The data used are the data obtained by simulation.

\section{Conflicts of Interest}

The authors declare that they have no conflicts of interest.

\section{Acknowledgments}

This study was supported by the National Key Research and Development Program of China (grant no. 2019YFB1706101) and Chongqing Technology Innovation and Application Demonstration Project (grant no. cstc2018jszX-cyzdX0143).

\section{References}

[1] A. R. Nia, M. H. Far, and S. T. A. Niaki, "A fuzzy vendor managed inventory of multi-item economic order quantity model under shortage: an ant colony optimization algorithm," International Journal of Production Economics, vol. 155, pp. 259-271, 2014.

[2] Z. Zhang, Xu Wang, Q. Guo, Z. Li, and Y. Wu, "Simulation and analysis of the complex behavior of supply chain inventory system based on third-party logistics management inventory model with no accumulating of unsatisfid demand," Complexity, vol. 2019, Article ID 3194093, 18 pages, 2019.

[3] H. L. Lee, V. Padmanabhan, and S. Whang, "Bullwhip effect in a supply chain," Sloan Management Review, vol. 38, pp. 93$102,1997$.

[4] K. P. Lin, P.-T. Chang, K.-C. Hung, and P.-F. Pai, "A simulation of vendor managed inventory dynamics using fuzzy arithmetic operations with genetic algorithms," Expert Systems with Applications, vol. 37, no. 3, pp. 2571-2579, 2010.

[5] J. W. Forrester, Industrial Dynamics, MIT Press, Cambridge, MA, USA, 1961.

[6] D. R. Towill, "Dynamic analysis of an inventory and order based production control system," International Journal of Production Research, vol. 20, no. 6, pp. 71-687, 1982.

[7] S. M. Disney, M. M. Naim, and D. R. Towill, "Development of a fitness measure for an inventory and production control system," in Proceedings of the 2nd International Conference on Genetic Algorithms in Engineering Systems: Innovations and Applications, Glasgow, UK, 1997.

[8] S. M. Disney, M. M. Naim, and D. R. Towill, "Genetic algorithm optimisation of a class of inventory control systems," International Journal of Production Economics, vol. 68, no. 3, pp. 259-278, 2000.

[9] S. M. Disney and D. R. Towill, "A discrete transfer function model to determine the dynamic stability of a vendor managed inventory supply chain," International Journal of Production Research, vol. 40, no. 1, pp. 179-204, 2002.

[10] J. Dejonckheere, S. M. Disney, M. R. Lambrecht, and D. R. Towill, "Measuring and avoiding the bullwhip effect: a control theoretic approach," European Journal of Operational Research, vol. 147, no. 3, pp. 567-590, 2003.

[11] P. Lin and D. S.-H. Wong, "Controller design and reduction of bullwhip for a model supply chain system using z-transform analysis," Journal of Process Control, vol. 14, pp. 487-499, 2004.

[12] T. Nagatani and D. Helbing, "Stability analysis and stabilization strategies for linear supply chains," Physica A: Statistical Mechanics and Its Applications, vol. 335, no. 3-4, pp. 644-660, 2004.

[13] S. M. Disney, "Supply chain aperiodicity, bullwhip and stability analysis with Jury's inners," IMA Journal of Management Mathematics, vol. 19, no. 2, pp. 101-116, 2008.

[14] E. Mosekilde and J. L. Laugesen, "Nonlinear dynamic phenomena in the beer model," System Dynamics Review, vol. 23, no. 2-3, pp. 229-252, 2007.

[15] X. Wang, S. M. Disney, and J. Wang, "Stability analysis of constrained inventory systems with transportation delay," European Journal of Operational Research, vol. 223, no. 1, pp. 86-95, 2012.

[16] C. A. Garcia, A. Ibeas, and R. Vilanova, "A switched control strategy for inventory control of the supply chain," Journal of Process Control, vol. 23, no. 6, pp. 868-880, 2013.

[17] X. Wang, S. M. Disney, and J. Wang, "Exploring the oscillatory dynamics of a forbidden returns inventory system," International Journal of Production Economics, vol. 147, pp. 3-12, 2014.

[18] J. Ma and F. Si, "Complex dynamics of a continuous bertrand duopoly game model with two-stage delay," Entropy, vol. 18, no. 7 , p. 266, 2016.

[19] F. Si and J. Ma, "Complex dynamics in a triopoly game with multiple delays in the competition of green product level," International Journal of Bifurcation and Chaos, vol. 28, no. 2, Article ID 1850027, 2018.

[20] X. Zhan, Q. Zhang, and W. F. Xie, "Dynamic performance and stability research of VMI-APIOBPCS in apparel industry based on control theory," International Journal of Enterprise Information Systems, vol. 14, no. 2, pp. 56-76, 2018.

[21] M. Z. Jin and L. J. Song, "Simulation analysis of production decision optimization of supply chain system based on nonlinear system and fractional differential operator," CHAOS, vol. 29, no. 1, Article ID 013129, 2019.

[22] J. Lin and M. M. Naim, "Why do nonlinearities matter? The repercussions of linear assumptions on the dynamic behaviour of assemble-to-order systems," International Journal of Production Research, vol. 57, p. 6824, 2019.

[23] X. Xu and S. D. Lee, "Management and optimization of chaotic supply chain system using adaptive sliding mode control algorithm," International Journal of Production Research, 2020.

[24] S. John, M. M. Naim, and D. R. Towill, "Dynamic analysis of a WIP compensated decision support system," International Journal of Manufacturing System Design, vol. 1, no. 4, pp. 283-297, 1994.

[25] Z. Li and G. Yan, "Analysis of complex dynamics behaviors in constrained supply chain system under uncertain demand process," Control and Decision, vol. 31, no. 1, pp. 173-179, 2016. 